(C) 2021, The Authors. Published by Elsevier Inc. and Fass Inc. on behalf of the American Dairy Science Association ${ }^{\circledR}$. This is an open access article under the CC BY-NC-ND license (http://creativecommons.org/licenses/by-nc-nd/4.0/).

\title{
Use of Lactobacillus paracasei isolated from whey for silver nanocomposite synthesis: Antiradical and antimicrobial properties against selected pathogens
}

\author{
Railean-Plugaru Viorica, ${ }^{1 *}$ Pomastowski Pawel, ${ }^{1}$ and Buszewski Boguslaw ${ }^{1,2}$ \\ ${ }^{1}$ Centre for Modern Interdisciplinary Technologies, Nicolaus Copernicus University in Toruń, Wileńska 4, 87-100 Toruń, Poland \\ ${ }^{2}$ Department of Environmental Chemistry and Bioanalytics, Faculty of Chemistry, Nicolaus Copernicus University in Toruń, Gagarina 7 , \\ 87-100 Toruń, Poland
}

\section{ABSTRACT}

The present research emphasizes the use of safe, inexpensive, and available whey using Lactobacillus paracasei as a source in silver nanocomposite synthesis as an alternative bioactive agent for dairy and biomedical applications. Through the multiinstrumental approach used in this study based on spectroscopic and microscopic methods as well as spectrometric techniques, the characterization and evaluation of silver composites and their antimicrobial and antiradical properties were enabled. Synthesized silver nanocomposites have been found in form of nanocrystals, naturally coated by an organic surface with high antimicrobial and antiradical properties. Furthermore, this work also presents an innovative approach regarding the organic surface (naturally secreted by the bacteria isolated from whey) of the core of nanoparticles, which has already been explored and therefore is starting to supplement the scientific approach concerning biologically synthesized nanoparticles. This work also presents a general frame on the resistance subject by performing the trial interaction of commercially available antibiotics (kanamycin and ampicillin) with new bioactive compounds that can create novel knowledge on complementing their action. Moreover, synthesized silver nanocomposites have shown great antioxidant and antimicrobial effects against various foodborne pathogens from dairy products and drug resistance pathogens found in the medical area to rank on the top of mortality rate.

Key words: Lactobacillus paracasei, silver composites, organic surface, antibacterial activity, biosynthesis

Received June 8, 2020.

Accepted October 16, 2020.

*Corresponding author: viorica.railean@umk.pl

\section{INTRODUCTION}

The development of the biological method seems to be a simple and viable alternative to chemical and physical methods. It has been reported that bionanoparticles provide opportunities to modify the inhibition of a specific metabolic pathway at the cellular level (Lok et al., 2006). Moreover, the bioactive complex has various nutraceutical and pharmaceutical applications. Obtaining a stable biocomplex is an essential step in the process of antiseptic and dietary supplement research, as well as their potential application to the pharmaceutical field. Therefore, the development of new safe agents or the modification of available ones to improve their effectiveness against drug-resistant microorganisms is a high priority.

On the other hand, the incorrect processing or storage of dairy products can be a source of extremely harmful microorganisms (Proteus mirabilis, Acinetobacter baumannii, Pseudomonas aeruginosa) to human health (Hassan and Frank, 2011; Dhanashekar et al., 2012; Saad et al., 2018). Milk ingredients also provide excellent nutrients for bacteria growth. Beside nutrients, milk is as well a source of healthy microorganisms such as lactic acid bacteria (LAB) strains, aimed to protect the human gastrointestinal tract against various infections. In addition to that, it is used in silver nanoparticle synthesis representing a great antimicrobial agent (Singh et al., 2015; Adebayo-Tayo and Popoola, 2017).

Currently, a considerable number of studies, including our works, refer to silver nanoparticles because of their multitude of advantages; that is, in addition to their antibacterial effect against a broad range of microorganisms (bacteria, fungus, parasites), they usually do not exert a cytotoxic effect toward eukaryotic cells or LAB strains. Since these aspects are very important when associated with the emergence of pathogenic bacterial strains possessing resistance toward several antibiotics, it is essential to point out that even the antimicrobial properties of drug silver nanoparticles synthesized by 
different bacteria vary from one bacterium to another (Chernousova and Epple, 2013; Singh et al., 2015; Deng et al., 2016; Railean-Plugaru et al., 2016a; Buszewski et al., 2017). Moreover, it has been demonstrated that the synergistic effect of silver nanoparticles and antibiotics presents a significantly higher effect when compared with antibiotics alone (Deng et al., 2016; Rogowska et al., 2017). Nevertheless, for other tested bacterial strains the combinations of biologically synthetized silver nanoparticles with antibiotics did not significantly change their efficacy compared with antibiotics and nanosilver alone (Buszewski et al., 2017).

Additionally, it is essential to underline that the use of the bacterial strain as a source for silver nanocomposite (AgNC) synthesis plays a crucial role. In fact, many studies have shown not only the size and concentrations of silver nanoparticles on antimicrobial effects but also the sources used for the synthesis processes are directly linked to the bacterial strain (Chauhan et al., 2013; Singh et al., 2015; Abd-Elnabya et al., 2016; Garmasheva et al., 2016). In the same order of ideas, the results presented by our group have demonstrated the efficient synthesis of nontoxic bioactive silver composites and presented in detail the use of dairy products (Lactococcus lactis strain) as a eco-friendly, inexpensive, and accessible precursor for biosilver composites in comparison to, for example, the Actinomycetes strain (Railean-Plugaru et al., 2017).

Furthermore, in light of increasing ecological problems, even with new applications appearing on the horizon, the future will definitely be focused on sustainable synthesis development of silver nanoparticles and their use in food and pharmaceutical fields. Certainly, the pursuit of new safe sources of silver composite synthesis, considering the present needs and without compromising future generations, will be of high interest. One suitable solution is the use of LAB strains from milk and products resulting from the processing of milk (e.g., whey).

Therefore, the purpose of this study first was to design a safe source for AgNC synthesis using bacterial strains isolated from whey and on the another hand to use the synthesized nanocomposites against harmful microorganisms found in dairy products and multidrugresistant strains in the medical area as well. Simultaneously, the present research has also been focused on a detailed analysis of the physicochemical properties of the synthesized AgNC, allowing the collection of a maximum amount of information on the bioparticle composites and the influence of these and antibiotic systems on the antimicrobial and antioxidant effects. A series of spectroscopy and microscopy methods and spectrometric techniques were applied. Moreover, the present research will summarize the comparison infor- mation of the synthesized silver nanoparticles (physicochemical properties, antimicrobial activity, coat organic core) using different bacterial strains as a source and also supplement the present knowledge concerning nanoparticle synthesis.

\section{MATERIALS AND METHODS}

\section{Synthesis of Lactobacillus paracasei ssp. paracasei Strain LPC20 Silver Nanocomposites}

The Lactobacillus paracasei ssp. paracasei strain LPC20 (LBPC) used in the present study for the synthesis of AgNC is a LAB strain from the collection of the Centre for Modern Interdisciplinary Technologies, Nicolaus Copernicus University in Toruń; this strain has been isolated from sweet whey $(\mathrm{pH}=5.9$; Drzycim, Poland). Isolation of the LPC20 strain was carried out using method described previously by Railean-Plugaru et al. (2017) with suitable modifications. In the present research, De Man, Rogosa and Sharpe agar medium has been used for isolating the necessary strain, and once the bacteria was isolated, the MALDI Biotyper Compass platform (Bruker Daltonik GmbH, Bremen, Germany) was used for the identification of the isolated strain. The de Man, Rogosa, and Sharpe agar medium has been chosen for the present study due to the optimal growth conditions for the lactobacilli; the respective culture medium is designed to favor the luxuriant growth of lactobacilli for laboratory study. The procedure for identification was performed according to the manufacturer's protocol, based on both raw and main spectra as described by Pomastowski and Buszewski (2019). Then, the Lactobacillus paracasei LPC20 isolated was deposited, under deposit no. B/00287, in the Polish Collection of Microorganisms.

The LBPC-AgNC synthesis involved 2 steps: the first step was the cultivation of bacterial strain followed by the synthesis. First, the isolated LBPC strain was cultivated in de Man, Rogosa, and Sharpe broth (SigmaAldrich, Poznan, Poland) medium and incubated (100 $\mathrm{rpm}$ ) at $26^{\circ} \mathrm{C}$ for $2 \mathrm{~d}$ in bioreactor Biostat A (Sartorius Stedim Biotech, Goettingen, Germany). Then, the obtained growth was centrifuged $(9,961 \times g$ at room temperature for $15 \mathrm{~min}$ ) and filtered (Durapore@ $0.22 \mu \mathrm{m}$ polyvinylidene difluoride membrane, Merck, Warsaw, Poland), and the filtered supernatant was combined with $3 \mathrm{mM} \mathrm{AgNO}$ (final concentration); the obtained mixture was directly centrifuged $(20,784 \times g$, room temperature, $10 \mathrm{~min}$ ) and the supernatant was placed for another incubation at $26^{\circ} \mathrm{C}$ in a shaker for $2 \mathrm{~d}$ in the dark. The produced bioactive nanocomposites (NC) were centrifuged at $20,784 \times g$ at room temperature for 30 min to concentrate. Then, they were purified 5 times 
by centrifugation at $20,784 \times g$ at room temperature for $15 \mathrm{~min}$ with water. Additionally, to remove unreacted silver ions and low molecular weight metabolites, a 3 -d period of dialysis was performed $(3 \mathrm{kDa}$ cut-off, Spectrum Labs, St. Paul, MN). The mixture of de Man, Rogosa, and Sharpe broth medium with $3 \mathrm{mM} \mathrm{AgNO}$ (final concentration) served as the control.

\section{LBPC-AgNC Biosilver Composite Characterization}

Electron Microscopy. The morphology of the biologically synthesized silver nanoparticles was studied using electron microscopy techniques. Compositional and conformational analysis of elemental silver presence was performed through energy dispersive X-ray spectroscopy (XFlash 4010, Bruker AXS) using scanning electron microscopy (LEO 1430VP) by fixing the silver powder particles on the microscope holder (conducting carbon strip). For the transmission electron microscopy (TEM; FEI Tecnai F20 X-Twin) analysis, the biologically synthesized silver nanoparticles were dropped on the carbon-coated copper grids and kept for solvent evaporation, before measurements. Selected area electron diffraction pattern and fast Fourier transform analysis (XFlash 4010 Bruker AXS) of the synthesized nanoparticles were also performed. The crystalline nature of $\mathrm{AgNC}$ was recorded by the powder X-ray diffraction (XRD) technique. The data were processed using the ORIGIN Pro/2016 software (OriginLab Corporation, Northampton, MA) and based on generated $2 \theta$ values, the $\mathrm{d}$-spacing and crystalline size values were determined. The interplanar distance and crystallite size were calculated using Bragg's and Debye-Scherrer equations, respectively (Railean-Plugaru et al., 2017).

Dynamic Light Scattering Analysis. Dispersion stability and size distribution profiles were measured by the dynamic light scattering (DLS) methods using the Zetasizer Nano Series (Malvern Instruments, Malvern, UK). The samples were dissolved in a $0.87 \% \mathrm{wt} / \mathrm{vol}$ $\mathrm{KCl}$ solution at $\mathrm{pH} 7$, then sonicated $20 \mathrm{~min}$ and analyzed using UV grade cuvettes for the hydrodynamic size distribution of the synthesized AgNC and folded capillary cells for the zeta potential investigation. Additionally, the synthesized silver NC have been investigated after period of time: initial time, and 0, 1, 2, 3, 4, 5 , and 6 d. Each measurement was carried out 3 times using standard red laser set at $633 \mathrm{~nm}$ wavelength by a dynamic instead of diffraction (DLS) approach. The data were expressed as \pm standard deviation.

Fourier Transform Infrared Spectroscopic Analysis. The possible presence of organics onto or into synthesized AgNC was investigated using the thin layer method in a DirectDetect Infrared Spectrometer (Merck Millipore, Darmstadt, Germany) by dropping an aliquot of the sample on the assay-free sample card (catalog no. DDAC00010-81). The samples were dissolved in distillated water and then the infrared spectrum was registered in a range of 1,350 to $1,870 \mathrm{~cm}^{-1}$, a range characteristic for the protein secondary structure (amide I and amide II) describing the repetitive conformations of proteins and peptides.

Fluorescence Spectroscopy. The obtained AgNC, previously sonicated (10 $\mathrm{min})$, were analyzed in a commonly used $10-\mathrm{mm}$ path length quartz cuvette. Threedimensional excitation-emission spectra were recorded using a spectrofluorometer Jasco FP-XX (Tokyo, Japan) in a range of 250 to $585 \mathrm{~nm}$ (excitation) and 260 to $600 \mathrm{~nm}$ (emission). The detection was made at $90^{\circ}$ to the incident beam with a high-voltage detector fixed to $800 \mathrm{~V}$. Relative fluorescence quantum yields were evaluated considering water as blank solvent.

MALDI-TOF/TOF-MS Analysis. The MALDITOF/TOF-MS system in our research has been used for both (1) identification of LAB strain, applied for the synthesis step, and (2) characterization of the synthesized AgNC. The samples preparation and MALDI acquisition for (1) has been performed in accordance with the procedure described by Pomastowski et al. (2019), with the suitable modifications, whereas the sample analysis of AgNC (2) was done by the dried droplet method, previously described by Pomastowski et al. (2015). In case of (1) the method was modified and no matrix was used, whereas in the case of (2) Q-cyano-4-hydroxycinnamic acid was used as a matrix at concentration $10 \mathrm{mg} / \mathrm{mL}$. In case of the second part of the assay (2), the identification of protein sequencing and the isotopic distribution of the synthesized $\mathrm{NC}$ were recorded based on mass spectra of 1- and 2-dimensional MS profiles; 3 separated plots were investigated. The MALDI-TOF/TOF-MS measurements were operated in positive reflection mode in the $\mathrm{m} / z$ range of 100 to 3,500 using a mass spectrometer (Bruker Daltonik $\mathrm{GmbH}$ ). The laser was set at $355 \mathrm{~nm}$ wavelengths and $2 \mathrm{kHz}$ frequency and the fingerprint spectra have been scanned at $25 \mathrm{kV} ; 2,000$ shorts were accumulated for one spectrum. The system was externally calibrated with the mixture of peptide calibration standard, protein calibration standard I and 2 signals characteristic for the matrix $[\mathrm{M}-\mathrm{H}]^{+}$and $[2 \mathrm{M}-\mathrm{H}]^{+}$, according to the standardized Bruker sample preparation procedure. The calibration has been performed in the range of 100 to $3,000 \mathrm{~m} / z$ with In last fit value of $32.88 \mathrm{mg} / \mathrm{L}$ and well fitted to the cubic enhanced model. For first (1) and second part (2) of the assay, the FlexControl and FlexAnalysis software (Bruker) were employed for the acquisition, processing, and visualization of data.

Antiradical Properties of LBPC-AgNC Using 2,2-Diphenyl-1-Picrylhydrazyl Assay. The 
antiradical properties of LBPC-AgNC and reference substances (trolox and rutin) were assessed based on the method described by Brand-Williams et al. (1995) with suitable modifications. Methanolic solution of $0.6 \mathrm{~m} M$ 2,2-diphenyl-1-picrylhydrazyl (DPPH) was mixed with different concentrations $(0.04,0.08,0.15$, $0.30,0.60$, and $1.20 \mathrm{mM}$ ) of each reductant (Red) at a ratio of $1: 1$. Once the samples were prepared, after 30 min of incubation in the dark $\left(23 \pm 1^{\circ} \mathrm{C}\right)$, the absorbance was recorded spectrophotometrically at $517 \mathrm{~nm}$. All obtained data were processed and expressed as a percentage of DPPH remaining at $30 \mathrm{~min}$ (1), DPPH scavenging effects (2), effective concentration (EC50) required to reduce the initial concentration of DPPH by $50 \%$ (3), antioxidant reducing power, antiradical power (ARP; 4), stoichiometry value (SchVal; 5) amount of Red required theoretically to reduce $100 \%$ of DPPH (EC100; the number of DPPH moles reduced by $1 \mathrm{~mol}$ of Red; 6) (Table 1). Methanol mixed with samples (1:1) served as blank control solutions. All results were recorded and presented as mean values \pm standard deviation of 3 repetitions.

\section{Antimicrobial Potential and Viability Approach}

For this purpose, different methodologies evaluating antimicrobial activity in vitro were applied. The classical MIC and well diffusion methods for the evaluation of AgNC efficacy were performed with fluorescence microscopy assay for visualization of bacteria viability under selected stress conditions.

Proteus mirabilis (ATCC25933), Acinetobacter baumannii (ATCC BAA-1605), Pseudomonas aeruginosa (ATCC 10145), Staphylococcus epidermidis (ATCC49461), Micrococcus luteus (ATCC 10240), Lactococcus lactis (ATCC 11454), and Lactobacillus casei (ATCC 334), acquired from Pol-Aura (Różnowo, Poland), and Saccharomyces cerevisiae (accession number: MG012794), purchased from the collection of Centre for Modern Interdisciplinary Technologies Nicolaus Copernicus University in Toruń, were used. The following antimicrobial agents: biologically synthesized LBPC2649-AgNC composites, 2 antibiotics [kanamycin (KAM; aminoglycoside) and ampicillin (AMP; $\beta$-lactamase), Sigma-Aldrich], and combinations of them, were investigated against the selected bacterial strains enumerated above.

Investigation of MIC of Biologically Synthesized LBPC2349-AgNC. A susceptibility test was performed based on the method recommended by Clinical and Laboratory Standards Institute (2006) guidelines, with suitable modifications. The colony suspension $\left(1 \times 10^{6} \mathrm{cfu} / \mathrm{mL}\right)$ prepared in Mueller-Hinton
Table 1. Calculation formulas of antiradical properties according to Brand-Williams et al. $(1995)^{1}$

\begin{tabular}{lc}
\hline Formula & Number \\
\hline DPPH remaining, $\%=\mathrm{A}_{\text {Red }} / \mathrm{A}_{D P P H} \times 100$ & {$[1]$} \\
DPPH scavenging effects, $\%=$ & {$[2]$} \\
$\left(\mathrm{A}_{D P P H}-\mathrm{A}_{\text {Red }}\right) / \mathrm{A}_{D P P H} \times 100$ & {$[3]$} \\
$\mathrm{EC} 50=$ remaining $\mathrm{DPPH} \%$ as a function & \\
of $[\mathrm{Red}] /[\mathrm{DPPH}]$ & {$[4]$} \\
$\mathrm{ARP}=1 / \mathrm{EC} 50$ & {$[5]$} \\
$\mathrm{SchVal}=\mathrm{EC} 50 \times 2$ & {$[6]$} \\
EC100 $=1 /$ SchVal & \\
\hline
\end{tabular}

${ }^{1} \mathrm{~A}_{\text {Red }}=$ absorbance of reductant (Red) and $\mathrm{A}_{D P P H}=$ initial absorbance of 2,2-diphenyl-1-picrylhydrazyl (DPPH) measured at $517 \mathrm{~nm}$; EC50 $=$ effective concentration; ARP $=$ antiradical power; SchVal $=$ stoichiometry value; $\mathrm{EC} 100$ = amount of Red required theoretically to reduce $100 \%$ of DPPH.

broth (Sigma-Aldrich) medium and antimicrobial solution of LBPC2349-AgNC were mixed in a ratio of 1:1 in a 96-well round-bottom plate (Sigma-Aldrich). The LBPC2349-AgNC solution was dispersed in the previously cultured bacteria and adjusted to an inoculum density of $1 \times 10^{6} \mathrm{cfu} / \mathrm{mL}$ with the following final concentrations: $100,50,25,12.5,6.25$, and 3.125 $\mu \mathrm{g} / \mathrm{mL}$. The suitable modifications of the Clinical and Laboratory Standards Institute method on the inhibition process of the tested bacterial strains under the LBPC2349-AgNC treatment were added to each well of the In Vitro Toxicology Assay Kit, Resazurin based (Sigma-Aldrich) that allows the determination of the metabolic activity of living cells expressed by the MIC value. The changes of the resazurin color from blue to pink determined the viability of cells. All measurements were prepared in triplicate. The data are expressed as \pm standard deviation and processed by Microsoft Excel (Microsoft Corp., Redmond, WA) using the bubble chart model according to the Microsoft Office support 2010 .

Investigation of Effectiveness of Antimicrobial Agents Using Well Diffusion Method. Tryptic soy agar plates were used for the evaluation of AgNC efficacy by the standard screening well diffusion method. For this step, the antimicrobial agents were used separately (antibiotic, LBPC-AgNC) and in combination (LBPC-AgNC + antibiotic). The concentration/well of investigated combinations was the sum of the antimicrobial agent concentrations separately. For the respective assay the following concentrations were used: antimicrobial agents alone: KAM, $5 \mu \mathrm{g} /$ well, and AMP, $20 \mu \mathrm{g} /$ well; LBPC-AgNC, $12.5 \mu \mathrm{g} /$ well; and antimicrobial agent combinations: LBPC-AgNC/AMP, $32.5 \mu \mathrm{g} /$ well, and LBPC-AgNC/KAM, $17.5 \mu \mathrm{g} /$ well. The antimicrobial agents were aseptically placed onto plates previously inoculated with $100 \mu \mathrm{L}$ of bacterial suspension. The diameter of the inhibition zones was 


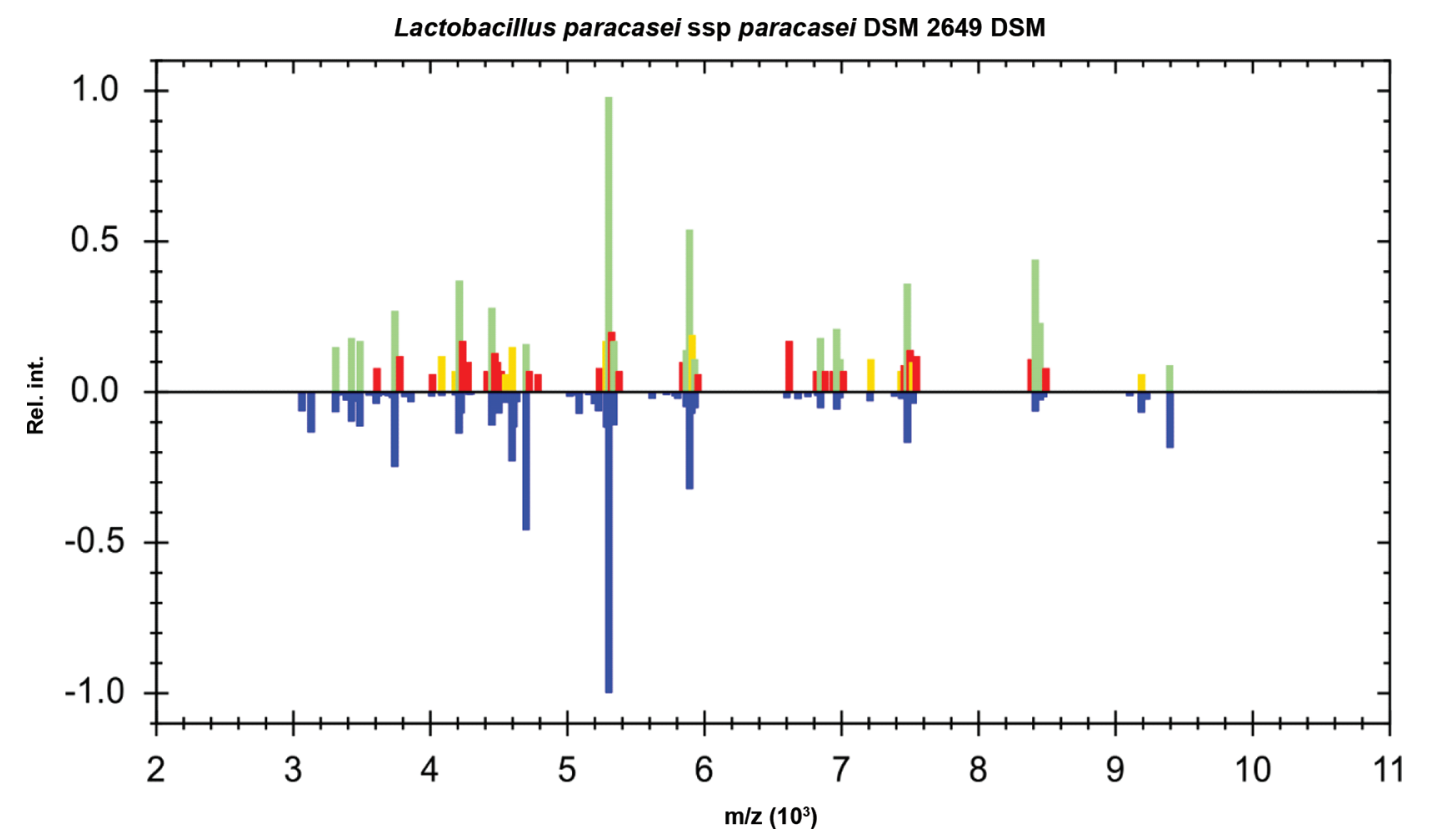

Figure 1. Main spectra and pick list of the isolate and Lactobacillus paracasei DSM 2649 reference strain identified by MALDI Biotyper Compass platform (Bruker Daltonik GmbH, Bremen, Germany). Green color = signal of the sample match with reference spectra of probability higher than $85 \%$, yellow $=$ signal of sample match with probability lower than $85 \%$, red $=$ no match. Rel. int. $=$ relative intensity.

measured in millimeters after $18 \mathrm{~h}$ of incubation at $35^{\circ} \mathrm{C}$. All investigations were made 3 times on identical samples. The data were expressed in terms of \pm standard deviation and processed by the Microsoft Excel program using the radar chart model according to the Microsoft Office support 2010.

Investigation of Antimicrobial Efficiency of LBPC-AgNC Using Fluorescence Microscopy Assay. Once the MIC value was found to be low, a higher concentration in the fluorescence microscopy assay was chosen to be suitable for investigation of the capacity of the synthesized LBPC-AgNC as an efficient antimicrobial agent. By this method, the viability of Staphylococcus epidermidis and Acinetobacter baumannii cells exposed to a concentration above the MIC of LBPC2349-AgNC was investigated. The cells $\left(1 \times 10^{6}\right.$ $\mathrm{cfu} / \mathrm{mL}$ ) were treated with $\mathrm{AgNC}$ and incubated for $24 \mathrm{~h}$ at $35^{\circ} \mathrm{C}$. Next, they were stained with 2 fluorochromes, acridine orange $(0.12 \mu \mathrm{g} / \mathrm{mL})$ and ethidium bromide $(0.4 \mu \mathrm{g} / \mathrm{mL})$, to distinguish the live and dead cells. The untreated Staphylococcus epidermidis and Acinetobacter baumannii cells served as the control to determine the background levels of viability under the experimental conditions. Microscopic images of specimens were recorded after $3 \mathrm{~h}$ and $24 \mathrm{~h}$ of incubation using an Axio Observer D1 (Zeiss, Oberkochen, Germany) fluorescence microscope under $43 \mathrm{He}$ (excitation $550 / 25$, emission $605 / 70$ ) and 38 (excitation $470 / 40$, emission 520/50) filters.

\section{RESULTS AND DISCUSSION}

\section{Identification of Lactobacillus paracasei ssp. paracasei LPC20}

The MALDI-TOF/TOF-MS system led to the identification of Lactobacillus paracasei ssp. paracasei by using the MALDI Biotyper Compass platform based on raw and main spectra. The score values for the LAB isolated were found to be more than 1.999 (2.03), classified as a high confidence level. The identified sequence overlaps with the most similar Lactobacillus paracasei ssp. paracasei DSM 2649 (Figure 1).

\section{LBPC-AgNC Biosilver Composite Characterization}

Synthesized AgNC were characterized by a complex of multiinstrumental techniques. The particularities of LBPC-AgNC synthesized by isolate, Lactobacillus paracasei ssp. paracasei were investigated by spectroscopy, spectrometry, and spectrofluorometric methods.

For the size investigation of LBPC-AgNC biocomposites, 3 different approaches were applied: DLS that allowed us to determine the hydrodynamic size distribution of biocolloids (nanocomposites) in DLS mode, TEM to determine the size of metal silver nanoparticles (metallic core), and XRD for crystallite size. According to the DLS method, 2 dominant populations were observed ranged from 75 to $194 \mathrm{~nm}$ (Figure 2A), with 
zeta potential value (Figure 1B) equal to $-30.16 \pm 1.76$ $\mathrm{mV}$. The intensity of the second signal, representing the second population, was higher than the intensity of the first signal (Figure 1A). This fact suggests that the predominantly hydrodynamic size of biocolloids is about $194 \mathrm{~nm}$. This particle size distribution remained constant for $3 \mathrm{~d}$. After this time, the size increased around $490 \pm 15.02 \mathrm{~nm}$ and the zeta potential slightly decreased to $-25 \pm 1.12 \mathrm{mV}$. Therefore, with increasing size of biocolloids in the hydrodynamic system, zeta potential value slightly decreased. However, the dispersion stability of the system was found to be still stable.

Based on energy dispersive X-ray spectroscopy and TEM results (Figure 3A), first the presence of metallic form of silver nanoparticles was proven, with binding energies in a range of 2.7 to $3.2 \mathrm{keV}$ characteristic for silver, and second their size was determined. The average size of metallic silver nanoparticles, according to TEM results, was found to be around $18 \pm 2.4 \mathrm{~nm}$. Moreover, the XRD (Figure 3D) and selected area electron diffraction pattern (Figure 3E) confirmed the cubic crystalline structure of biologically obtained nanoparticles with an average crystallite size equal to $8.03 \pm 2.4 \mathrm{~nm}$ and d-spacing of $0.2394 \mathrm{~nm}$.

The $2 \theta$ signals found $\left(38.27^{\circ}, 44.30^{\circ}, 64.65^{\circ}, 77.55^{\circ}\right.$, $81.66^{\circ}, 111.04^{\circ}$, and $\left.114.97^{\circ}\right)$ correspond to the (111), (200), (220), (311), (222), (331), and (420) Bragg reflection numbers, respectively; coordinated with the
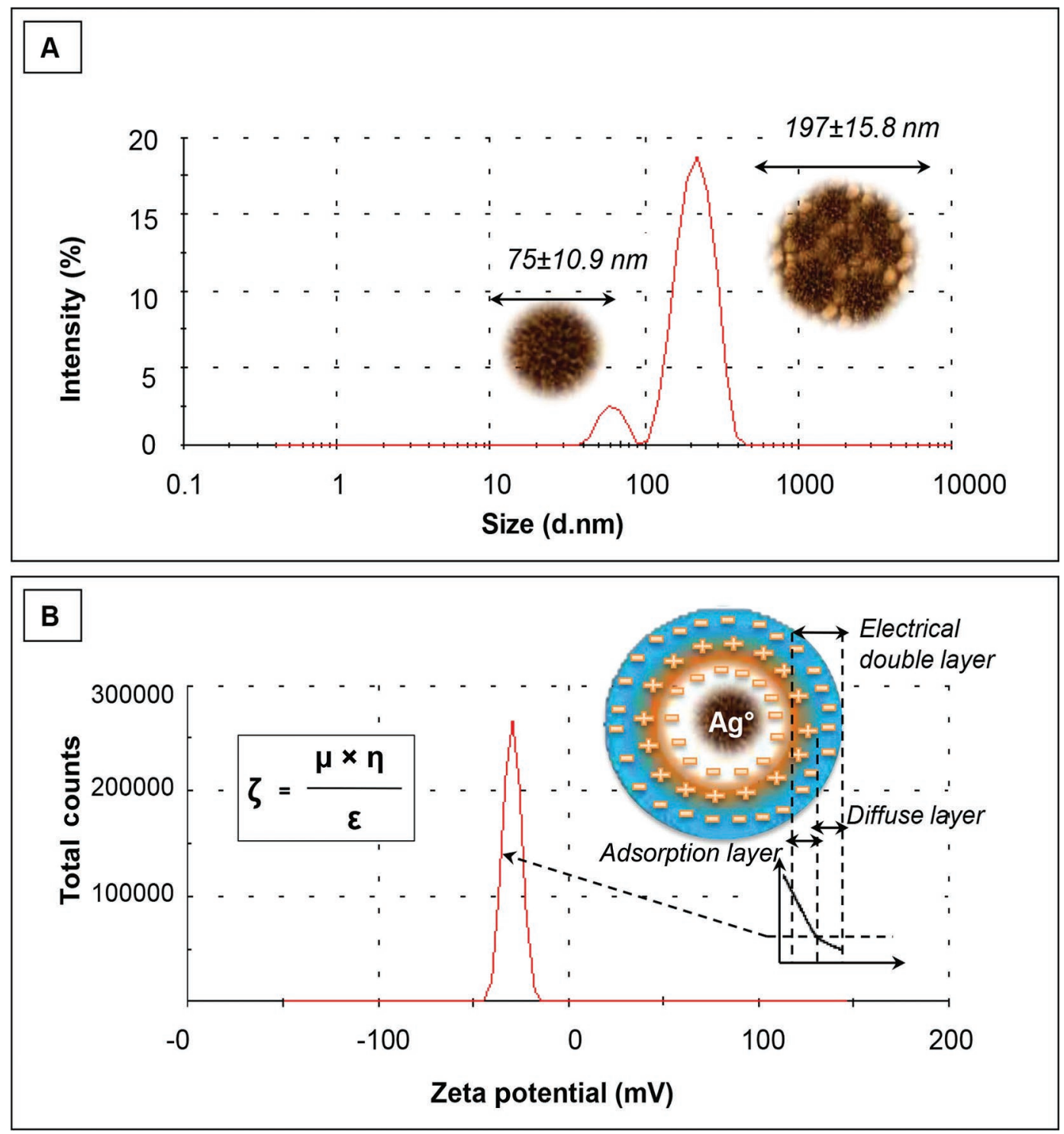

Figure 2. Size (A) and zeta potential (B) distribution of synthesized Lactobacillus paracasei ssp. paracasei (LBPC)-silver nanocomposites (AgNC). $\varepsilon=$ dielectric constant; $\eta=$ solution viscosity; $\mu=$ electrophoretic mobility; $\zeta=$ zeta potential $(\mathrm{mV})$. 

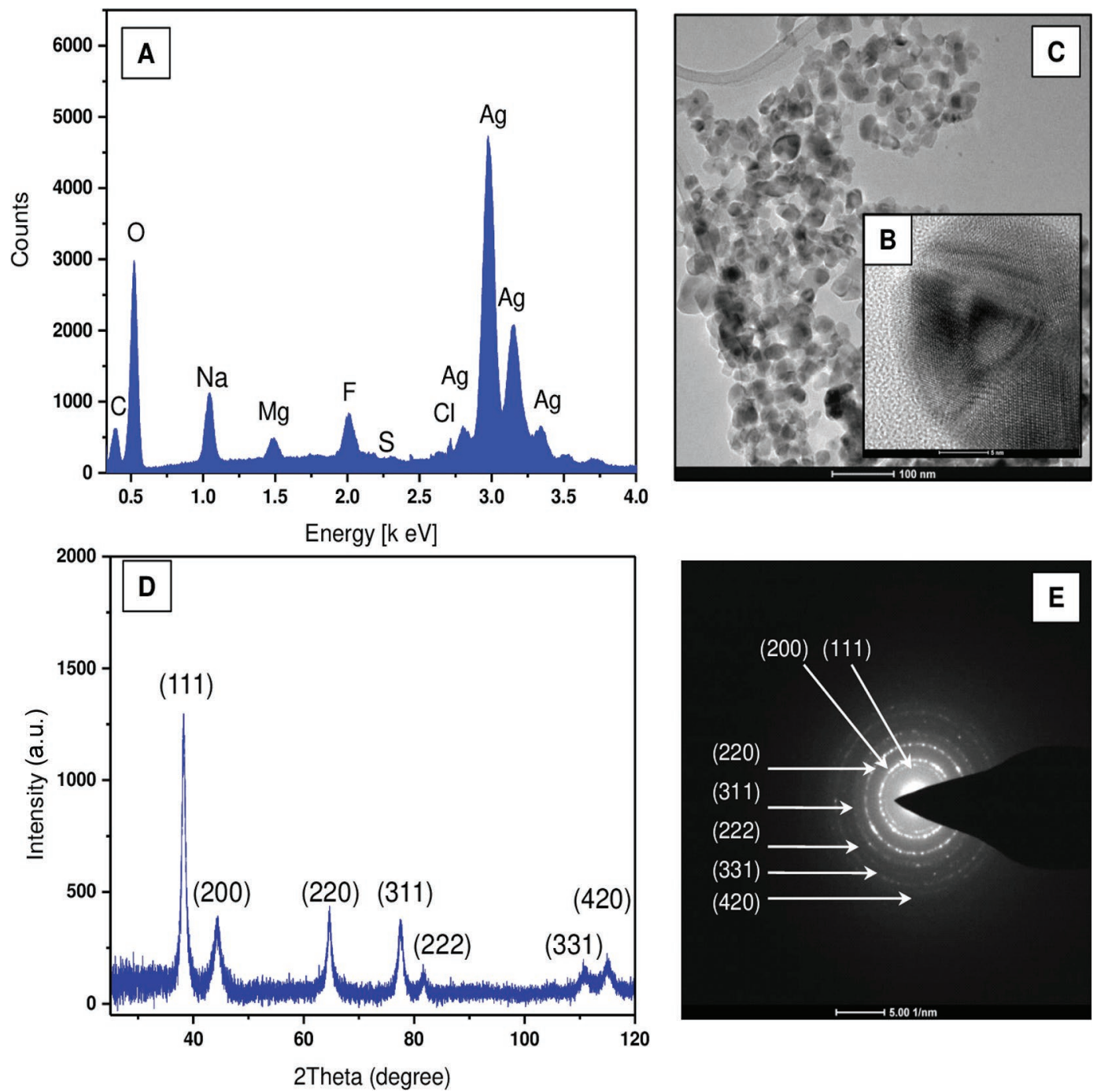

Figure 3. The particularities of Lactobacillus paracasei ssp. paracasei (LBPC)-silver nanocomposites (AgNC) synthesized as presented by energy dispersive X-ray spectrum (A), transmission electron micrograph (B), fast Fourier transform image (C), X-ray diffraction (D), and selected area electron diffraction $(\mathrm{E})$ patterns. a.u. = arbitrary units.

lattice planes of the face-centered cubic silver crystals (Ag/XRD, reference no. 00-003-0921; Jung, 1926). The observed differences in the silver size distribution, recorded by XRD, TEM, and DLS techniques, depends on the method used; the XRD method allowed for the determination of crystallite size, the TEM technique for the size of silver nanoparticles, and DLS generated the hydrodynamic size value of the complex nanostructure of the AgNC, defined as biocolloids. From this fact we deduce that silver nanoparticles are composed of several crystallites "bonded" to each other, consequently generating a higher size value of the silver nanoparticles presented as a cluster (TEM results), and on the other hand, higher hydrodynamic size value of the AgNC (DLS results). This phenomenon also suggests the pres- ence of organic compounds represented a surface of biomolecules for capping and stabilizing the metallic silver, either through the electrostatic attraction of negatively charged molecules (carboxyl groups) or through free amine groups (Railean-Plugaru et al., 2017).

In addition to the already mentioned information, it should be specified that the synthesized AgNC have been additionally characterized by the spectroscopy approach, proving repeatedly the organic layer located onto or into the bioactive silver nanoparticle core. Due to the fact that this technique is widely used, the implementation of the infrared spectrum for the biocolloids granted us the access to the identification of vibrations, which are assigned to the functional groups responsible for the interaction with metallic silver or silver cations. 
Therefore, the Fourier transform infrared spectra (Figure 4) illustrate the conformation of noticed functional groups registered in the range of 1,350 to $1,870 \mathrm{~cm}^{-1}$, a range characteristic for amide vibration (amide I and amide II), which are the main bands of the protein infrared spectrum mainly associated with the binding and stretching vibration directly related to the backbone conformation. According to the literature, the signal at $1,451 \mathrm{~cm}^{-1}$ originates from the aromatic $\mathrm{C}=\mathrm{C}$ stretching vibrations, whereas the signal found in the 1,548 $\mathrm{cm}^{-1}$ region assigns to the binding vibration of amide $\mathrm{II}$, predominantly $\nu \mathrm{C}-\mathrm{N}$ vibration bands (Venyaminov and Kalnin, 1990; Barth and Zscherp, 2002). The signal noticed at $1,649 \mathrm{~cm}^{-1}$ indicates the presence of $\alpha$-helices in protein and polypeptide structures (Venyaminov and Kalnin, 1990), whereas $1,635 \mathrm{~cm}^{-1}$ is related to amide I group caused by stretching of $\beta$-sheet structure (Barth and Zscherp, 2002). Moreover, the same signal localized at $1,649 \mathrm{~cm}^{-1}$ is related to $\mathrm{C}=\mathrm{O}$ vibrations in Asn AA, whereas the signal registered at $1,635 \mathrm{~cm}^{-1}$ is assigned to stretching vibration of $\nu_{\mathrm{S}} \mathrm{CN}_{3} \mathrm{H}_{5}{ }^{+}$originating as well from Arg (Venyaminov and Kalnin, 1990). The presence of stretching vibration at $1,748 \mathrm{~cm}^{-1}$ is generated by the $\mathrm{C}=\mathrm{O}$ from the carboxylic acid group (Movasaghi et al.,
2008). The presence of branching of organic surface in NC structure has been reported by many authors (Bhat et al., 2011; Awwad et al., 2012; Mohanta and Behera, 2014). Those observations are also in agreement with our previous studies, proving the presence of different functional groups on the AgNC structure synthesized by different bacteria strains, namely Streptacidiphilus and Lactococcus lactis. Moreover, the shift of vibration frequencies depends on the type of the bacteria strain used for the synthesis (Railean-Plugaru et al. 2016a, 2017; Buszewski et al., 2018), and on the $\mathrm{pH}$ and affinity to various solvents (Railean-Plugaru et al., 2017, 2018; Buszewski et al., 2018).

This phenomenon is connected with the secretions of different metabolites produced by the bacterial strain isolated from whey, selected for the synthesis and protonation and deprotonation of functional groups located on the organic layer covering the metallic core of silver nanoparticles.

Molecules responsible for covering the metallic core of silver nanoparticles have also been characterized by spectrometric laser desorption ionization (LDI) approach in tandem with time-of-flight analyzer (LDI TOF-TOF-MS) as a complementary technique.

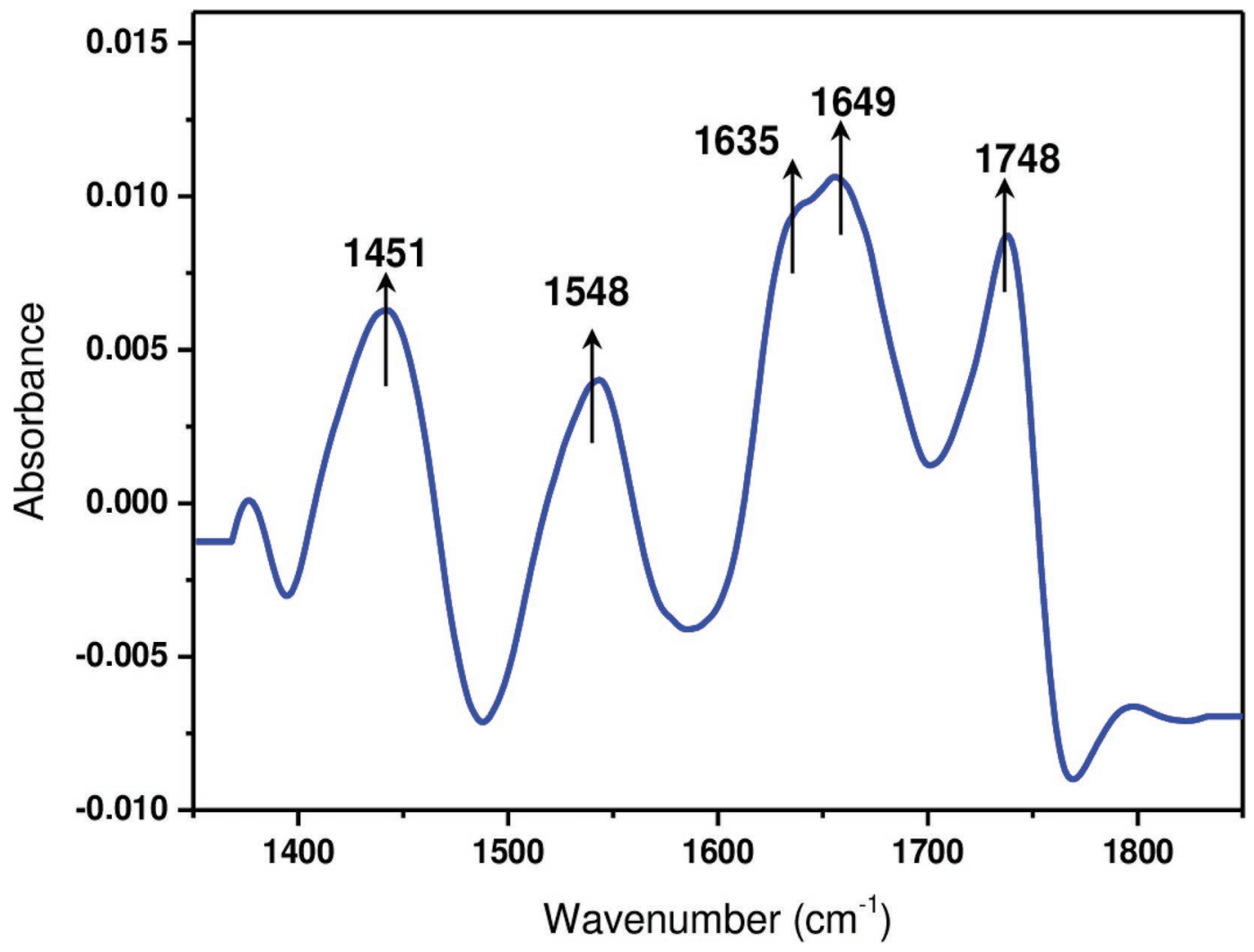

Figure 4. Fourier transform infrared spectra presenting conformation of functional groups onto or into Lactobacillus paracasei ssp. paracasei strain LPC20 DSM 2649 (LBPC2649)-silver nanocomposite (AgNC) structure registered in the range of 1,350 to $1,870 \mathrm{~cm}^{-1}$. 
Moreover, our previous work (Railean-Plugaru et al., 2018) has proven and identified the AA sequences by the $\alpha$-cyano-4-hydroxycinnamic acid-assisted LDI process in the organic layer covering the surface of silver nanoparticles, synthesized by L. lactis strain, justifying that particles are a uncommon cluster structure that may be described as being composed of a double silversilver $\left(\left[\mathrm{Ag}_{2}\right]^{+}\right)$and multi $\left(\left[\mathrm{Ag}_{\mathrm{n}}\right]^{+}\right)$combination with the organic layer. In the present research we used this approach without adding any matrix for the characteristics of AgNC synthesized by the Lactobacillus paracasei source. The 1- and 2-dimensional LDI analysis was performed. The obtained result provides the necessary potential for the application of biologically synthetized AgNP for nanostructure-assisted laser desorption and ionization (Pomastowski et al., 2019). This results from the surface plasmon resonance properties of synthetized AgNC. Metallic and nanostructured nanoparticles are charge donor for themselves and molecules bonded on their surface. The 1-dimensional LDI MS spectra (Figure 5A) show the molecular fingerprint of the synthesized AgNC recording the presence of silver cluster (Figure 5B-E) and organic deposits (coats; Figure $5 \mathrm{~F}-\mathrm{I})$ naturally covering the metal nanoparticles. Figure 5 illustrates not only the silver-organic connection but also records the simple silver isotopes and silver cluster presence; the same phenomenon was observed in the present study as in the case of the AgNC synthesized by the L. lactis strain, previously published by our group (Railean-Plugaru et al., 2018). Moreover, based on model isotopic patterns, silver $\left([\mathrm{Ag}]^{+}\right)$, silversilver $\left([\mathrm{Ag} 2]^{+}\right)$, and multi- $\left([\mathrm{Agn}]^{+}\right)$combinations with the organic layer were identified. As evidence, Figure 5B-E presents different isotopic distributions of silver depending on the number of silver atoms that compose the clusters with the $m / z$ generated at $m / z=107.149$ $[\mathrm{Agg}]^{+}, m / z=215.773[\mathrm{Ag} 2]^{+}, m / z=322.671[\mathrm{Ag} 3]^{+}$, $m / z=970.729[\mathrm{Ag} 7]^{+}, m / z=1,650.554[\mathrm{Ag} 13]^{+}$, and $m / z=2,050.422[\mathrm{Ag} 16]^{+}$. On the other hand, the application of laser ionization fragmentation technologies (LIFT) fragmentation approach led to the identification of peptides and AA sequences (Figure 5F-I). For instance, the signals registered at $\mathrm{m} / z=970.729, \mathrm{~m} / \mathrm{z}$ $=1,650.554, \mathrm{~m} / z=2,050.422$, and $\mathrm{m} / z=2,260.187$ suggest the presence of AA: Ile/Leu-Cys (I/L-C), Cysteine-Ile/Leu (Cys-I/L); AA i-types: asparagineArg-Ser (iN-iR-IS); and AA: Trp-Ser (W-S) sequences, respectively.

Moreover, the observations recorded by the spectroscopic method (Fourier transform infrared), which presents the sum of oscillation rotational bands, are in concordance with the spectrometry results (LDI LIFT MS), demonstrating first the presence of the organic coat and second the composition of the organic surface.
Moreover, the presence of asparagine and arginine AA registered at 1,649 and $1,635 \mathrm{~cm}^{-1}$, respectively, have also been recorded in composition of the organic coat that are connected with the silver core, registered by LDI LIFT MS. Therefore, it is noteworthy that the respective AA as well as the rest of the identified AA sequences are involved in coating naturally into or onto silver crystals and silver nanoparticles recorded by XRD and TEM techniques.

Based on the LIFT fragmentation, model isotopic pattern, and application of FlexControl software building blocks), the following formulas have been determined: C9H17Ag7N2O2S1 $(m / z=970.729), \quad \mathrm{C} 10 \mathrm{H} 21 \mathrm{Ag}-$ 13N2O3S1 $(\mathrm{m} / z=1,650.554), \mathrm{C} 10 \mathrm{H} 27 \mathrm{Ag} 16 \mathrm{~N} 7 \mathrm{O} 2(\mathrm{~m} / z$ $=2,050.422)$, and $\mathrm{C} 14 \mathrm{H} 16 \mathrm{Ag} 18 \mathrm{~N} 3 \mathrm{O} 3(\mathrm{~m} / z=2,260.187)$ with the following elemental composition: $\mathrm{C} 11.12 \%, \mathrm{H}$ $1.76 \%, \mathrm{Ag} 77.65 \%, \mathrm{~N} 2.88 \%$, O 3.29\%, S $3.30 \%(\mathrm{~m} / z=$ 970.729); C 7.27\%, H 1.28\%, Ag 84.90\%, N 1.70\%, O $2.9 \%, \mathrm{~S} 1.94 \%(\mathrm{~m} / z=1,650.554) ; \mathrm{C} 6 \%, \mathrm{H} 1.36 \%, \mathrm{Ag}$ $86.15 \%, \mathrm{~N} 4.89 \%$, O $1.60 \%(\mathrm{~m} / z=2,050.422)$; and $\mathrm{C}$ $7.70 \%, \mathrm{H} 0.74 \%$, Ag $87.72 \%$, N $1.92 \%$, O $1.92 \%(\mathrm{~m} / z$ $=2,260.187$ ).

It is notable that similar signals $(\mathrm{m} / z=970.729$ and $m / z=2,050.422$ ) recorded in the present study for AgNC synthesized by L. paracasei strain (LBPCAgNC) were also noticed for $\mathrm{AgNC}$ mediated by $L$. lactis isolate (LCLB56-AgCs), published previously by our group (Railean-Plugaru et al., 2018). By analyzing the LIFT fragmentation results of LBPC-AgNC and LCLB56-AgCs, different numbers of silver atoms connected with the organic part in the case of $\mathrm{m} / \mathrm{z}$ $=970.729$ (LCLB56-AgCs $[\mathrm{Ag}]^{+}$and LBPC-AgNC $\left.[\operatorname{Ag} 7]^{+}\right)$were observed, whereas in the case of $m / z=$ $2,050.422$, approximately the same number of silver atoms were noticed (LCLB56-AgCs $[\mathrm{Ag} 17]^{+}$and LBPC$\left.\mathrm{AgNC}[\mathrm{Ag} 16]^{+}\right)$. Moreover, different peptide sequences were recorded in both cases; $[\mathrm{AECys}-\mathrm{Cmc}-\mathrm{CcPP}-\mathrm{NP}]^{+}$ for LCLB56-AgCs and $[\mathrm{I} / \mathrm{L}-\mathrm{C}]^{+}$for LBPC-AgNC in the case of $m / z=970.729$, whereas [C-N] for LCLB56$\mathrm{AgCs}$ and $[\mathrm{iN}-\mathrm{iR}-\mathrm{iS}]^{+}$for $\mathrm{LBPC}-\mathrm{AgNC}$ in the case of $m / z=2,050.422$. However, in both cases the presence of organic coats was recorded in terms of AA and peptide sequences. It seems that organics secreted into the medium can first bind in or into silver core in different modes, and second the secreted metabolites depend on the sources used for the synthesis. These strategies and knowledge give a justification of the presence of metabolites produced by the L. paracasei strain during the inoculation step that are naturally biocoating the silver metallic core.

Additionally, the current work has supplemented these results with the fluorescence spectroscopy assay presenting 2 specific bands that registered the excitation $\left(\lambda_{\mathrm{ex}}\right)$ and emission wavelengths $\left(\lambda_{\mathrm{em}}\right)$. The $\lambda_{\mathrm{ex}} /$ 
$\lambda_{\text {em }}$ wavelength for LBPC23-AgCs was found to be 214 $\mathrm{nm}, 338 \mathrm{~nm}$ (I) and $270 \mathrm{~nm}, 538 \mathrm{~nm}$ (II), respectively. Figure $6 \mathrm{~A}$ shows the excitation $(214 \mathrm{~nm})$ and emission $(338 \mathrm{~nm})$ bands reflecting the effect of organics core, fluorescence of aromatic AA such as tryptophan, tyrosine, and phenylalanine (Ghisaidoobe and Chung, 2014), and Figure 6B shows the excitation (270 nm) and emission $(538 \mathrm{~nm})$ bands characteristic for silver light interaction to the core of nanocomposites (Siwach and Sen, 2009).

Taking into consideration that all of the results led to the confirmation of the natural presence of an organic surface generated by the bacteria isolated from the whey-protein/peptide components into or onto the silver nanoparticles core that are referred to as biocolloids. As is well known, the biocolloids are characterized by the negative charge on the particle surface in a dispersion medium. Therefore, the layer diffusion processes are influenced by the protonation and deprotonation state of biomolecules, often considered an es- sential fact that induces significant structural changes (Hunter, 1988).

Since the surface charge density is correlated with the zeta potential $(\zeta)$, accordingly, the value of the zeta potential varies depending on $\mathrm{pH}$, reflecting stability of the biocomplex dispersion. In this fact, our previous work described the influence of different $\mathrm{pH}$ on the surface charge of silver nanoparticles (Railean-Plugaru et al., 2016a, 2018; Buszewski et al., 2018). The most important aspect to mention is that when $\mathrm{pH}$ is increasing, more competition occurs between protons and metal ions for negatively charged binding sites. It has also been observed by other authors (Mohanta and Behera, 2014) that on the electrolyte ion concentration increment decreases the thickness of the electrical double layer as a result of nonspecific adsorption of ions, which determines the reduction in the value of the potential. Since it has been reported that zeta potential of colloids plays a significant role in many physico-chemical processes and depends on both the

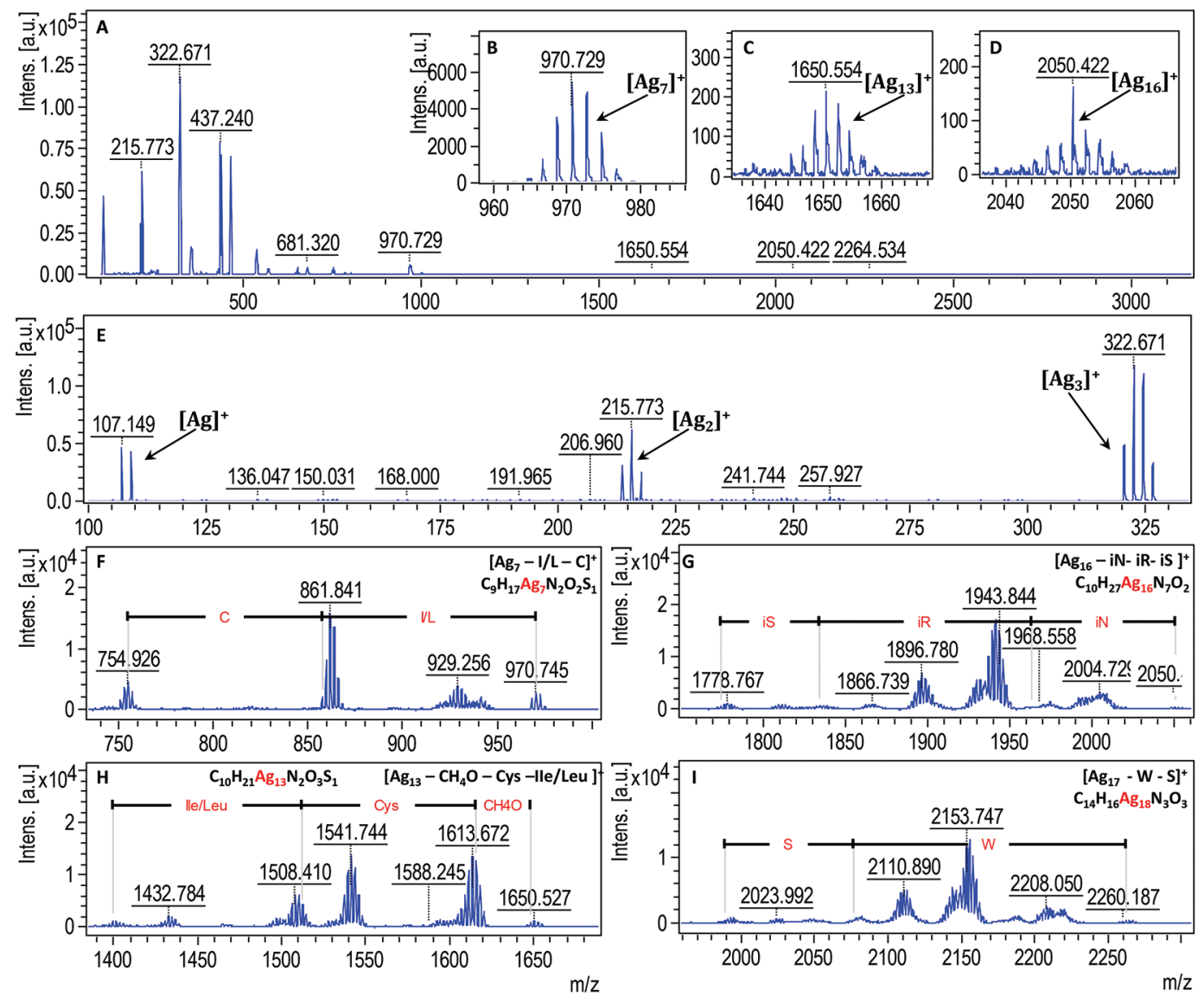

Figure 5. Mass spectra presenting the 1-dimensional (A-E) MALDI-TOF-MS of molecular fingerprint and isotopic distribution of Lactobacillus paracasei ssp. paracasei (LBPC)-silver nanocomposites ( $\mathrm{AgNC}$ ) and 2-dimensional metal-organic signals $(\mathrm{F}-\mathrm{I})$, where $\mathrm{C}=\mathrm{Cys}$; $\mathrm{I} / \mathrm{L}=\mathrm{Ile} / \mathrm{Leu}$; $\mathrm{S}=$ immonium ions of serine; $\mathrm{iR}=$ immonium ions of arginine, iN = immonium ions of asparagine; $\mathrm{S}=$ serine; $\mathrm{W}=$ tryptophan. Intens. = intensity; a.u. = arbitrary units. 
$\mathrm{pH}$ value as well as ionic strength conditions, in the present research the zeta potential measurements were carried out under the following optimal conditions: $\mathrm{pH}=7, \mathrm{KCl}(0.087 \%)$. Moreover, based on literature data (Bhat et al., 2011; Awwad et al., 2012; Mohanta and Behera, 2014; Garmasheva et al., 2016) and our previous studies (Railean-Plugaru et al., 2016a, 2018; Buszewski et al., 2018), it is notable to underline that it is not excluded that both zeta potential value as well as size of $\mathrm{AgNC}$ depend not only on the $\mathrm{pH}$ values but also on the secreted metabolites used by the bacteria strain as a source for the synthesis, consequently influencing the optical properties, hydrophobicity, or reactivity of biocolloids. Moreover, the stability of the dispersions, the phenomenon of aggregation, and silver complex adhesion are certainly additional contributions to the known information on the biological and antiradical activity of bioactive AgNC. Furthermore, in addition to the above enumerated characteristics, the silver $\mathrm{NC}$ has also been reported as a strong antiradical scavenger (Bhakya et al., 2016; Khorrami et al., 2018; Salari et al., 2019).

The free stable radical DPPH has been used in present study to determine the ARP of biologically synthesized $\mathrm{AgNC}$ as a potential Red. For comparative estimation of the ARP of NC, 2 well-known reference standards (trolox and rutin) were also investigated. The \% DPPH scavenging effects, efficient concentrations of Red decreasing the initial concentration of DPPH with 50\% (EC50), ARP, and number of moles of reduced DPPH have been determined based on the remaining DPPH (\%) at a fixed reaction time (30 $\mathrm{min})$. First, based on the remaining DPPH \%, the scavenging effects of tested reductants were determined. Figure 7A presents the plotted data of the decreasing of $\%$ $\mathrm{DPPH}$ in function of different Red concentrations. By analyzing the data obtained, it was observed that the DPPH scavenging behavior depends on both the nature of the Red as well as the concentration used; the tested reductants decreased the initial concentration of DPPH to $17.2 \pm 2.1(\mathrm{NC}), 5.08 \pm 1.7$ (rutin), and $4.75 \pm 1.5$ (trolox). Moreover, the standard references (rutin and trolox) presented similar behavior of DPPH reduction compared with synthesized NC. The same observations have been noticed for the \% DPPH scavenging effects. According to Mishra et al. (2012), this phenomenon can be associated with the classification of the NC Red as a slow Red; this means that total NC was unused on the DPPH scavenging at $30 \mathrm{~min}$. However, it was noticed that DPPH scavenging values increase with the increase of the Red concentration and DPPH scavenging effects of $\mathrm{NC}$ reach about $83 \% \pm 1.2$, whereas rutin and trolox reach about 95\%, not much less than standard references. Second, the EC50 and ARP of samples were evaluated from the plot of \% DPPH remaining as a function of molar ratio of Red to DPPH ([Red]/ [DPPH] ) (Figure 7B).

The effective concentration of AgNC to reduce $50 \%$ of initial concentration of DPPH was found to be 0.5 $\mathrm{m} M$, almost one time higher than the EC50 of refer-

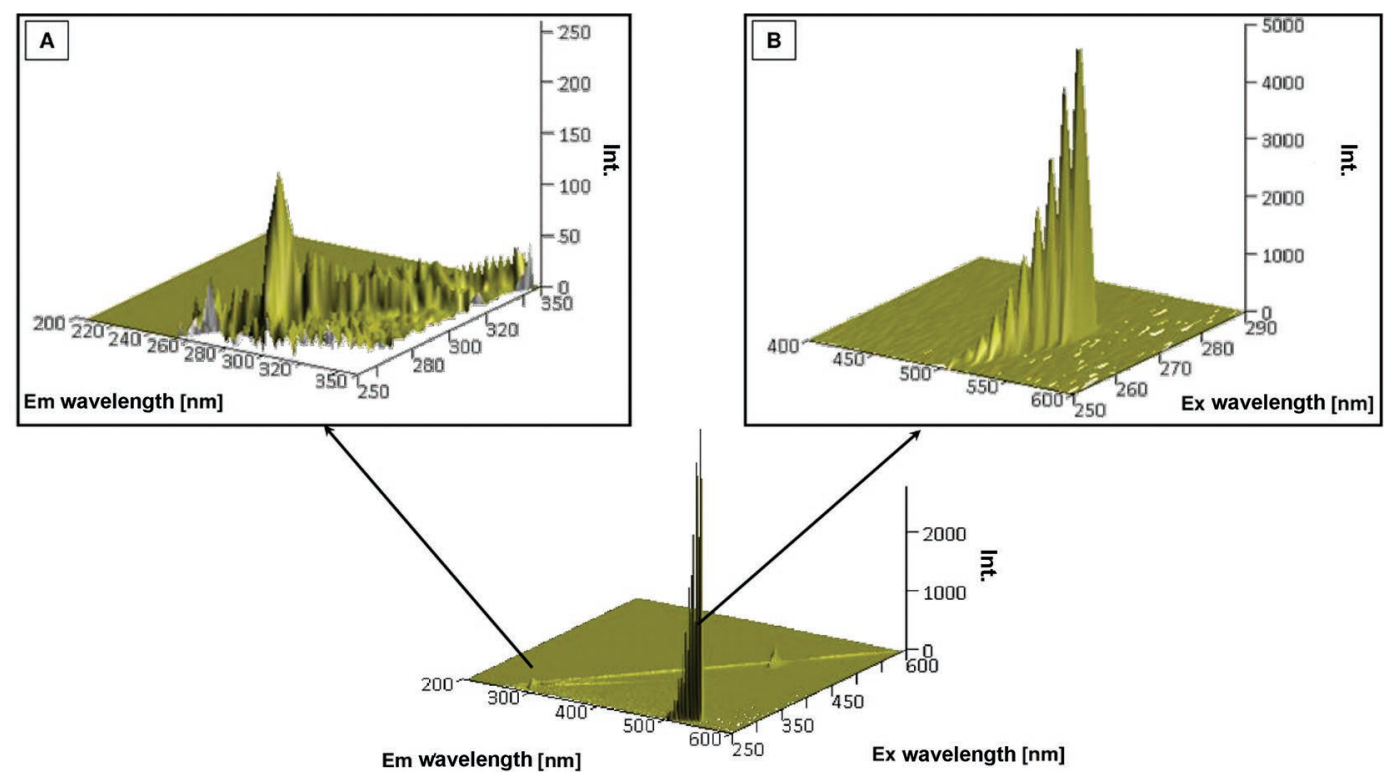

Figure 6. Fluorescence spectrum presenting the fluorescence effect of organic core (A) and silver nanoparticles (B) generated by the synthesized Lactobacillus paracasei ssp. paracasei strain LPC20 DSM 2649 (LBPC2649)-silver nanocomposites $($ AgNC). Em = emission; Ex = excitation; Int. = intensity. 

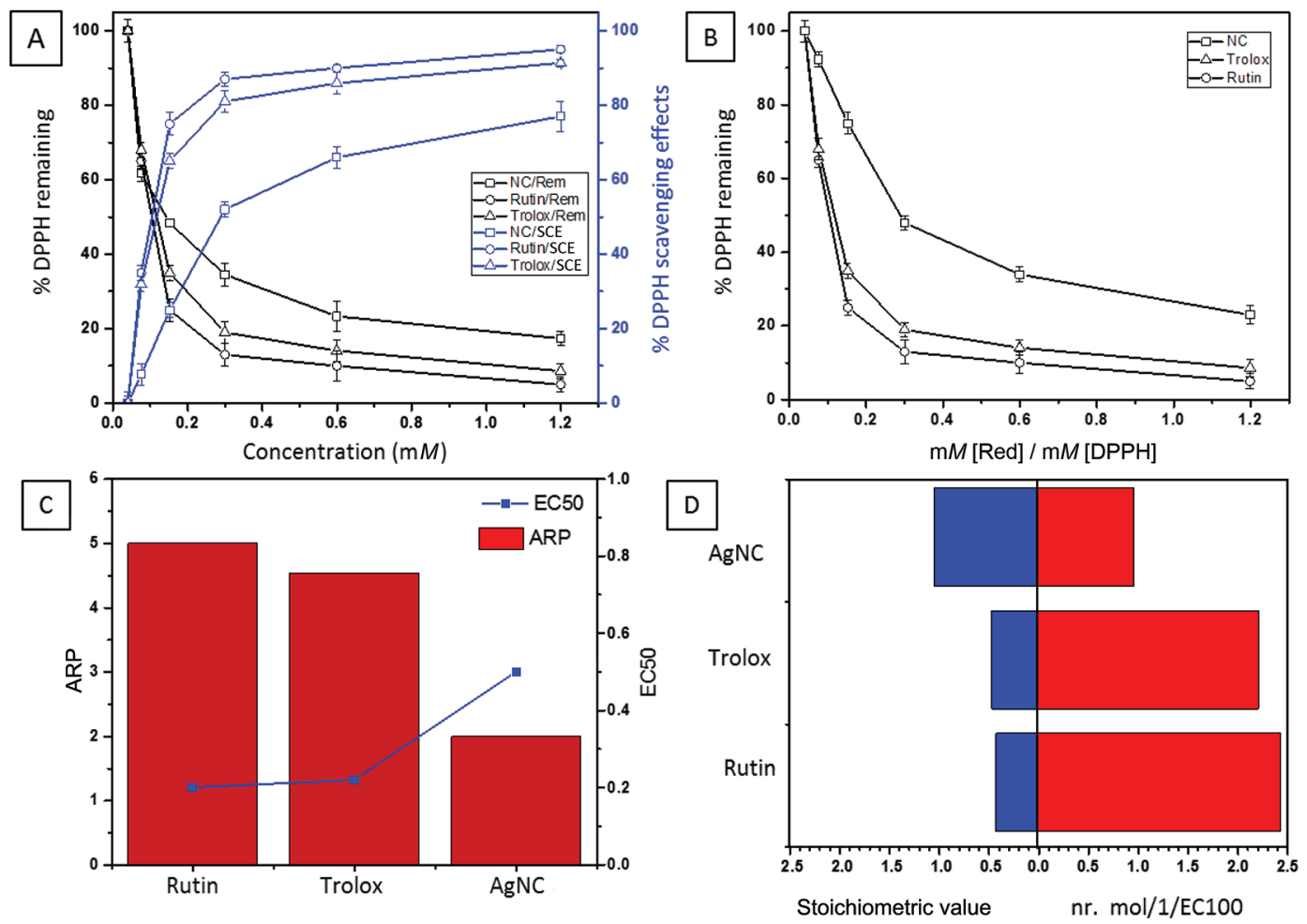

Figure 7. Plots presenting the percentage of 2,2-diphenyl-1-picrylhydrazyl (DPPH) scavenging effects (A), percentage of DPPH remaining as a function of $\mathrm{m} M[\operatorname{Red}] / \mathrm{m} M[\mathrm{DPPH}])$ (B), EC50 and ARP values (C), and stoichiometric value correlation to mole number of reduced DPPH (D). EC50 = effective concentration; ARP $=$ antiradical power; SchVal $=$ stoichiometry value; EC100 $=$ amount of reductant $($ Red $)$ required theoretically to reduce $100 \%$ of DPPH. $\mathrm{NC}=$ nanocomposites; Rem $=\%$ DPPH remaining; SCE $=\%$ DPPH scavenging effect.

ence reductants (rutin, $0.2 \mathrm{mM}$, and trolox, $0.22 \mathrm{mM}$ ) whereas ARP values, 2.00 (NC), 4.55 (trolox), and 5.00 (rutin), and ARP (NC) were about one time less then ARP values of reference reductants. However, the obtained data in the current study present a lower or almost the same EC50 value compared with the literature data where EC50 value has been reported in the range of 0.4 to $6 \mathrm{mM}$ (Bharathi and Bhuvaneshwari, 2019; Daphedar, 2020).

The predicted antiradical capacity of $\mathrm{NC}$ required to decrease the initial concentration of DPPH by $100 \%$ has also been determined and has been found to be as follows: $1 \mathrm{~mol}$ of $\mathrm{NC}$ is able to reduce $1 \mathrm{~mol}$ of DPPH, whereas $0.44 \mathrm{~mol}$ of trolox $-2.27 \mathrm{~mol}$ of DPPH and $0.4 \mathrm{~mol}$ of rutin $-2.50 \mathrm{~mol}$ of DPPH. Many researchers (Bhakya et al., 2016; Khorrami et al., 2018; Salari et al., 2019) have reported the antioxidant capacity of silver NC ranging from 0.13 to $45 \mathrm{mM}$ (expressed in $\mu \mathrm{g} / \mathrm{mL}$ ). What is noteworthy is that the antiradical capacity of AgNC depends on several parameters such as method used, DPPH concentration, reaction time, and reaction medium, and can be attributed not only to the interaction of the silver properties with the free radical but also to the sources (bacterial strain or plants) used for the synthesis that can secrete various metabolites (e.g., cysteine) during the synthesis process. Moreover, cysteine has been reported as a model antioxidant since Blois (1958), and once the MALDI-TOF-MS technique has recorded the presence of several AA in the organic core it is not excluded that the high antiradical capacity of AgNC synthesized in the present research is generated by the both metal silver nanoparticles as well as the organic surface.

\section{Antimicrobial Potential and Viability Approach}

The multidrug-resistant microorganisms infections have become a global concern in public health. Nowadays, this problem leads to higher hospitalization rates, higher healthcare costs and, consequently, to immunity reduction and increased mortality. Due to the fact that over the years bacteria have learned to become "immune" to the drug, even the spectacular widespread discovery of antibiotics does not stop or decrease bacterial diseases. The antimicrobial resistance mechanism is defined by different ways such as limitation uptake of drugs, inactivation or efflux of drugs, and modification of the drug target, depending on the drug used 
or microorganism tested. Pseudomonas aeruginosa ssp., Acinetobacter baumannii ssp., and Proteus mirabilis ssp. have been found to produce several types of enzymes (e.g., oxacillinases, extended spectrum beta-lactamases, extended-spectrum ampicillin $\mathrm{C}$ beta-lactamase, ampicillin $\mathrm{C}$ beta-lactamase, imipenemase, Verona Integronencoded metallo- $\beta$-lactamase) responsible for the resistance mechanism (Poirel et al., 2001; Bonnet et al., 2002). Therefore, to overcome the problem of drug resistance, a methodology of the synthesis of drugs that will not be able to generate feedback mechanisms is required.

To achieve the purposed goal, 3 different methodologies were involved: (1) the classical MIC, (2) well diffusion methods, and (3) fluorescence microscopic assay for visualization of bacteria viability under selected stress conditions. For this purpose, 3 multidrug-resistant gram-negative pathogens found in wound infections and even in the dairy industry, namely Proteus mirabilis (ATCC25933), Acinetobacter baumannii (ATCC BAA1605), and Pseudomonas aeruginosa (ATCC 10145); 2 gram-positive bacteria species, Staphylococcus epidermidis (ATCC49461) and Micrococcus luteus (ATCC 10240), that occur as normal microbiota of humans and mammalian skin, respectively, or are found in raw cow milk drawn aseptically from the udder mammalian skin (Micrococcus luteus; Bruno et al., 2009); 2 more gram-positive strains, Lactococcus lactis (ATCC 11454) and Lactobacillus casei (ATCC 334), with generally recognized as safe (GRAS) status by American Food and Drug Administration, and one yeast species Saccharomyces cerevisiae [accession number: MG012794] were used, found in cow milk as a result of nutritional yeast supplementation. Biologically synthesized LBPCAgNC composites, 2 antibiotics (AMP and KAM), and combinations of them (LBPC-AgNC/AMP and LBPC$\mathrm{AgNC} / \mathrm{KAM}$ ) were investigated for this purpose.

Figure $8 \mathrm{~A}$ and Table 2 present the bacterial segregation in terms of MIC of synthesized LBPC-AgNC composites. After $24 \mathrm{~h}$ of incubation, 3 multidrug-resistant pathogens, Pseudomonas aeruginosa, Acinetobacter baumannii, and Proteus mirabilis, and 2 strains, part of normal microbiota skin, Micrococcus luteus (mammalian) and Staphylococcus epidermidis (humans), presented more similarities and are recorded separately from the other safe strains, 2 LAB such as Lactococcus lactis and Lactobacillus casei, and Saccharomyces cerevisiae isolate. Moreover, Saccharomyces cerevisiae was found to present a higher MIC value $(3.12 \mu \mathrm{g} / \mathrm{mL})$ compared with Pseudomonas aeruginosa, Acinetobacter baumannii, Proteus mirabilis, Micrococcus luteus, and Staphylococcus epidermidis, where the MIC value was $1.56 \mu \mathrm{g} / \mathrm{mL}$. However, no relevant differences between respective MIC values were recorded; the MIC value of Saccharomyces cerevisiae was one time higher than the enumerated ones. Furthermore, no inhibitory effect against LAB bacterial strains (Lactococcus lactis and Lactobacillus casei) was noticed.

According to Figure 8B and Table 2, which present the full profile of all tested microorganisms treated with LBPC-AgNC, selected antibiotics alone (KAM and AMP) and their combinations (LBPC-AgNC/ AMP and LBPC-AgNC/KAM) based on diameter of inhibition zone values $(\mathrm{mm})$, dissimilarity between all investigated samples was observed and 4 main clusters were generated. High similarity between distribution of inhibition potential value of LBPC-AgNC and their combination with antibiotics (LBPC-AgNC/AMP and LBPC-AgNC/KAM) against Pseudomonas aeruginosa, Acinetobacter baumannii, Proteus mirabilis, and Micrococcus luteus was highlighted with the inhibition zone in the range of 15.5 to $18.0 \mathrm{~mm}$. On the other hand, no inhibition zone of antibiotics alone (AMP, KAM) against the same microorganisms was noticed.

Moreover, both antibiotics alone (AMP and KAM) as well as their combination with AgNC (LBPC-AgNC/ AMP and LBPC-AgNC/KAM) have been scattered together, in the same manner, only against safe microorganisms (Lactococcus lactis, Lactobacillus casei, and Saccharomyces cerevisiae).

In contrast to $\mathrm{AgNC}$ combinations with antibiotics, the LBPC-AgNC alone did not generate any inhibition zone against the same microorganisms, except the Saccharomyces cerevisiae strain that presented a slight inhibitory potential $(6 \mathrm{~mm} \pm 0.6)$, much less than in the case of LBPC-AgNC/AMP and LBPC-AgNC/KAM combinations where the inhibition zones were found to be in range of 20 to $31 \mathrm{~mm}( \pm 1.0)$.

This fact suggests that the antimicrobial properties of the respective combinations are generated by the antibiotics alone, once the AgNC alone did not present any inhibitory effect against respective safe microorganisms. It is important to highlight that in radar charts (Figure 8B), the antimicrobial potential values of all tested antimicrobial agents against Staphylococcus epidermidis are first scattered in an arbitrary manner than in the clusters discussed above in the case of other microorganisms, and second, the respective bacteria is susceptible to almost all tested antimicrobial agents except for KAM alone, presenting almost the same inhibition zones with the diameter about $19 \pm$ $1.0 \mathrm{~mm}$ for LBPC-AgNC, $23 \pm 1.2 \mathrm{~mm}$ for both AMP as well as LBPC-AgNC/AMP, and $20 \pm 1.0 \mathrm{~mm}$ for LBPC-AgNC/KAM. Kanamycin did not present an inhibition zone against the Staph. epidermidis strain. It is necessary to underline that no synergy effect was observed. The combinations of AgNC presented the same antimicrobial potential as antibiotics and $\mathrm{AgNC}$ alone. 
Therefore, this suggests a high inhibitory effect against Staph. epidermidis strain, once the antibiotics neither presented much higher antimicrobial effect nor improved the AgNC antimicrobial activity. Furthermore, the MIC values of the respective $\mathrm{NC}$ were found to be $1.56 \mu \mathrm{g} / \mathrm{mL}$ even against pathogen microorganisms and were not active against safe strains such as LAB strains. Additionally, the antimicrobial effect of LBPC-AgNC was also noticed and visualized in Figure 9. In contrast to control samples, after $24 \mathrm{~h}$ of incubation with
LBPC-AgNC $(12.5 \mu \mathrm{g} / \mathrm{mL})$, an increase of dead cells was observed in the case of both investigated strains. This phenomenon has proven once again the strong effect of biosynthesized AgNC. Moreover, the obtained $\mathrm{AgNC}$ in the present study showed a lower MIC value against Proteus mirabilis and Pseudomonas aeruginosa that are recognized as harmful microorganisms in the dairy sector than the LCLB56-AgCs synthesized by another LAB strain (Lactococcus lactis) where the MIC value was found to be 3.12 and $6.25 \mu \mathrm{g} / \mathrm{mL}$ for Proteus

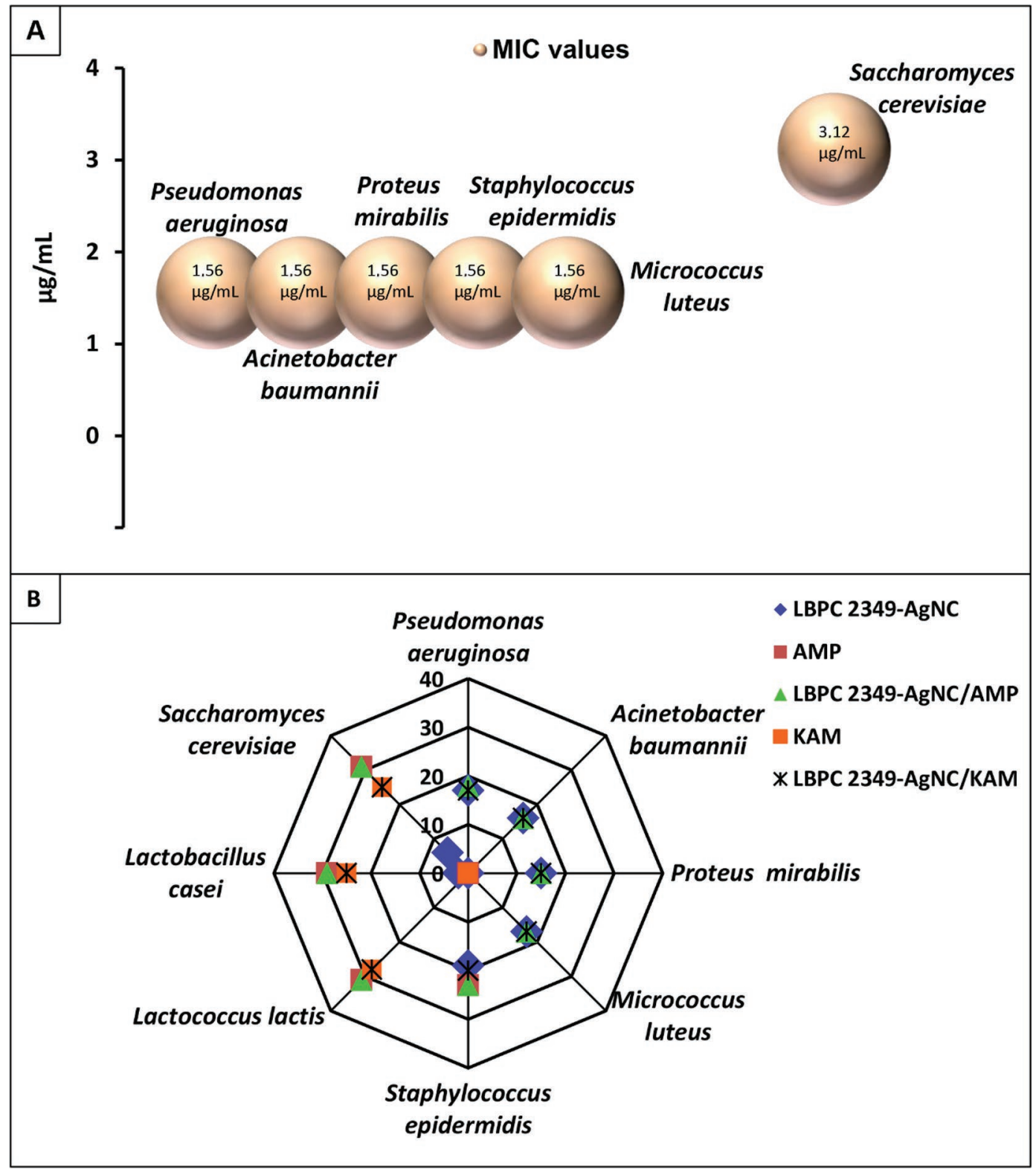

Figure 8. Bubble charts presenting the antimicrobial effect against tested microorganisms under silver nanocomposite (AgNC) stress expressed in MIC values (A) and radar charts presenting a full profile of tested microorganisms treated with synthesized AgNC, selected antibiotics alone, and their combinations, expressed in diameter of inhibition zone (mm; B). The AgNC did not show an inhibitory effect against Lactococcus lactis and Lactobacillus casei strains. $\mathrm{LBPC}=$ Lactobacillus paracasei $\mathrm{ssp}$. paracasei; $\mathrm{KAM}=\mathrm{kanamycin} ; \mathrm{AMP}=$ ampicillin. 
mirabilis and Pseudomonas aeruginosa, respectively (Railean-Plugaru et al., 2017). Furthermore, against the same bacterial strains, different AgNC (CGG and HGG) synthesized by Actinomycetes strains was investigated. In this case our group also found higher MIC values compared with the synthesized $\mathrm{AgNC}$ in the present study, using L. paracasei as a source (RaileanPlugaru et al., 2015; Buszewski et al., 2018). The obtained Ag_CGG11n and Ag_HGG16n nanocomposites showed a MIC value equal to $6.25 \mu \mathrm{g} / \mathrm{mL}$ against Proteus mirabilis and Pseudomonas aeruginosa except for Ag_HGG16n against Proteus mirabilis where the MIC value was much higher than Ag_CGG11n (synthesized by Actinomycetes), LCLB56-AgCs (synthesized by $L$. lactis), and even LBPC2649-AgNC (synthesized by $L$. paracasei).

The action mechanism of KAM consists of inhibition of protein synthesis, which is responsible for bacteria growth by binding to the bacterial $30 \mathrm{~S}$ ribosomal subunit, causing the misreading of tRNA while the AMP bind to the specific penicillin binding proteins by inhibition of cell wall synthesis, which in turn causes cell lysis. The mechanism action of antibiotics is well known, whereas that of AgNC is still a debated topic. However, one of the proposed mechanisms is the ability of silver $\mathrm{NC}$ to anchor to the cell wall causing damage in the cell membrane, in turn increasing its permeability (Prabhu and Poulose, 2012). On the other hand, many studies have reported the silver nanoparticles action via the "Trojan horse" mechanism, based on interaction of secondary released silver ions to cysteine by thiol groups (Hsiao et al., 2015). In addition, phospholipids portion of the bacterial membrane have also been found to be a site of action for the silver species (Dibrov et al., 2002).
Moreover, Lok et al. (2006) investigated the mode of antibacterial action of silver $\mathrm{NC}$ and silver ions, demonstrating a similar membrane-targeting mechanism of action, for what is more, the silver $\mathrm{NC}$ revealed to be more effective that silver ions. Additionally, they demonstrated an alteration in the expressions of a panel of envelope proteins (OmpA, OmpC, OmpF, OompA, MetA) and heat shock proteins, (IbpA, IbpB, and 30S ribosomal subunit $\mathrm{S} 6$ ). Therefore, it seems that the silver nanoparticles operate as an antibiotic and it cannot be ruled out that the high antimicrobial potential of $\mathrm{AgNC}$ noticed in the present study is a result of $\mathrm{AgNC}$ contact with the cell wall followed by the uncontrolled releasing of silver ions based on the "Trojan horse" mechanism, thus generating the expression changes of the respective proteins.

The given drug is a major problem in the treatment of microbial resistance. This problem is responsible for millions of deaths annually. Hence, it is obviously necessary to take measures to minimize the drug-resistant bacteria. However, in the case of positive result of antibacterial therapy, more danger may occur, namely yeast and fungal infections. In fact, various studies have reported applications of many types of silver nanoparticles against different microorganisms (Chauhan et al., 2013; Singh et al., 2015; Abd-Elnabya et al., 2016; Garmasheva et al., 2016).

According to these results and those previously published by our group (Railean-Plugaru et al., 2016a, 2017; Buszewski et al., 2017), it has been observed that antimicrobial activity of the biologically synthesized AgNC depends on the bacterial strain used for AgNC synthesis, and second, on the AgNC concentrations. The different enzymes (e.g., reductase) and metabolites

Table 2. Antimicrobial effect against tested microorganisms under silver nanocomposite stress, expressed as inhibition zone (mm), and MIC value

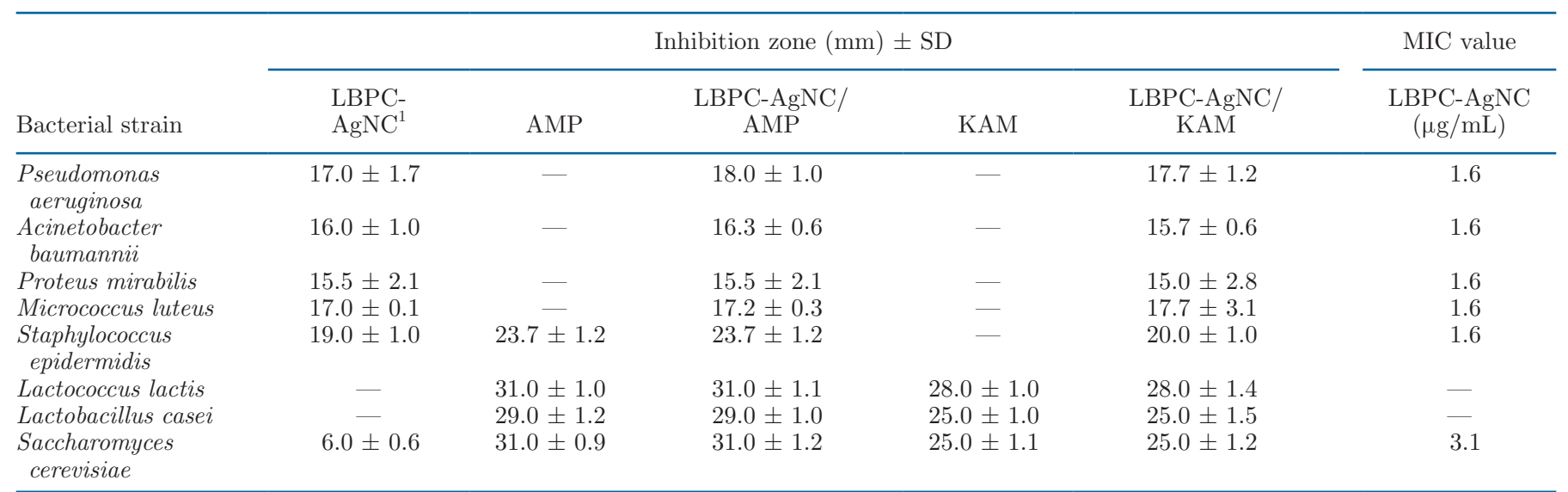

${ }^{1} \mathrm{LBPC}=$ Lactobacillus paracasei $\mathrm{ssp}$. paracasei strain LPC20; AgNC = silver nanocomposite; AMP = ampicillin; KAM = kanamycin. 


\section{Acinetobacter baumannii}
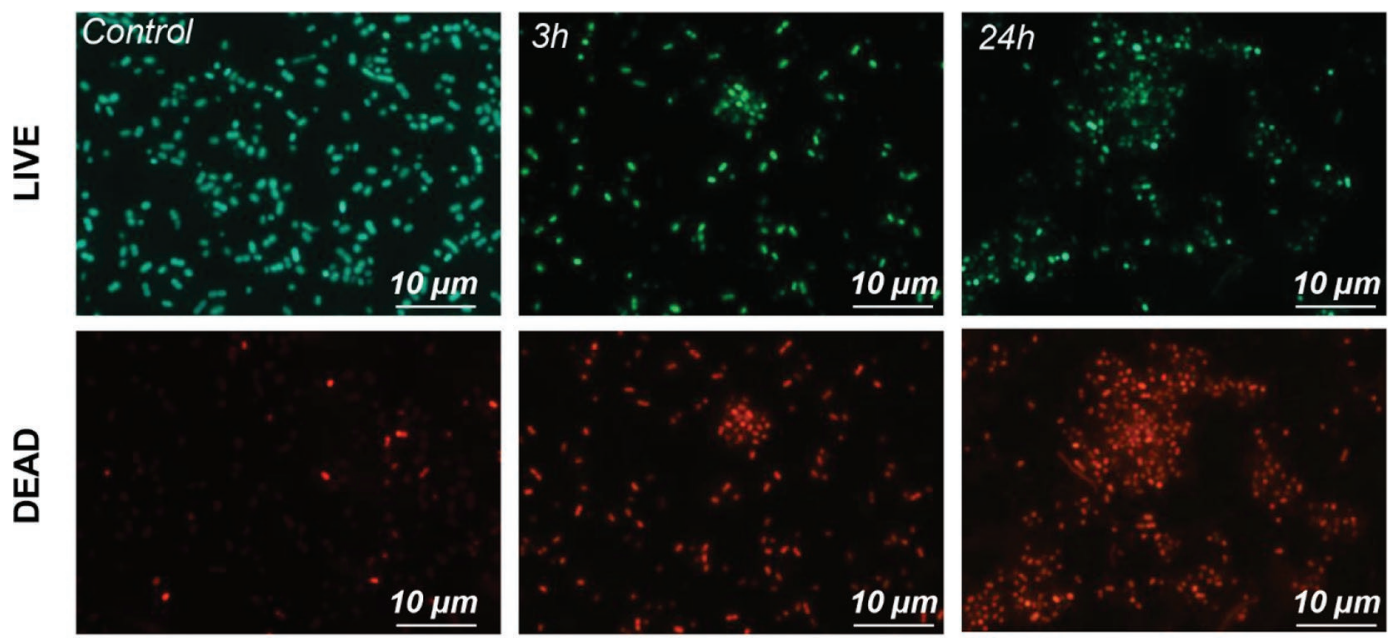

Staphylococcus epidermidis
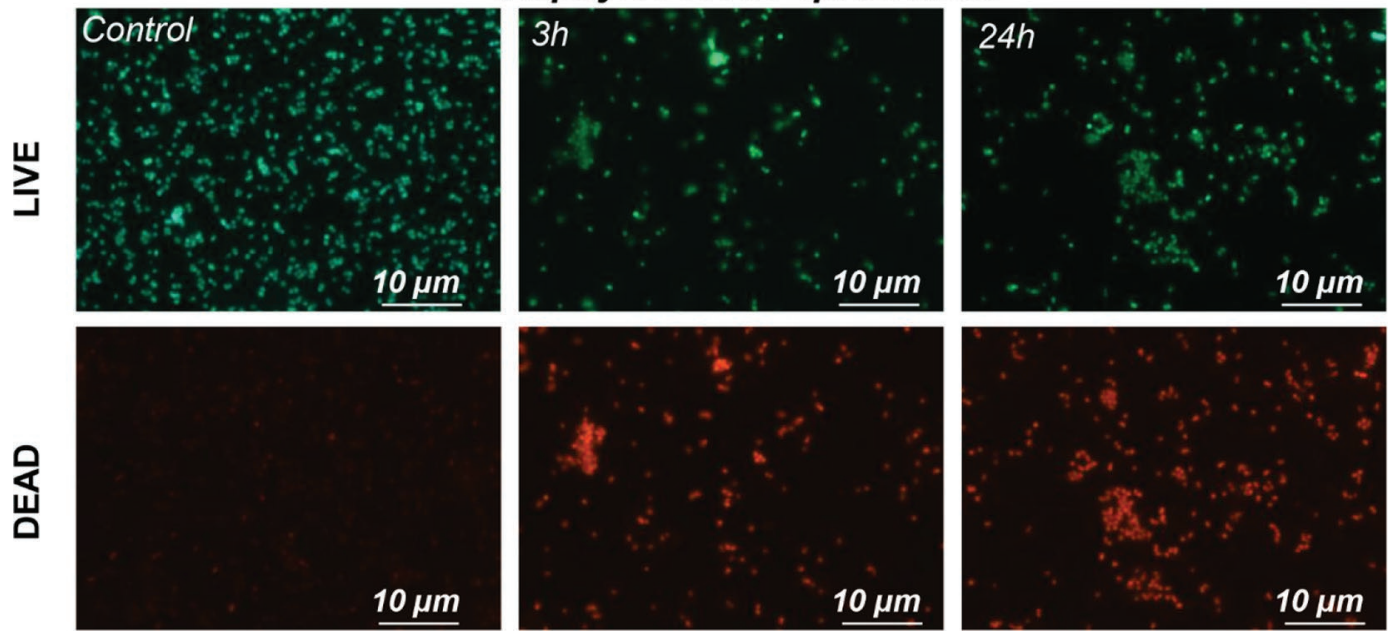

Figure 9. Viability imaging presenting the effect of Lactobacillus paracasei ssp. paracasei strain LPC20 DSM 2649 (LBPC2649)-silver nanocomposite (AgNC; $12.5 \mu \mathrm{g} / \mathrm{mL}$ ) against selected bacterial strains, where red represents dead cells and green represents live bacteria cells.

(AA, peptides, and their degradation products) of various bacterial strains [e.g., L. lactis (Railean-Plugaru et al., 2017) or Actinycetes (Railean-Plugaru et al., 2016a,b; Buszewski et al., 2017, 2018)] are secreted to the medium culture. Moreover, in addition to the antimicrobial effect, these components also influence the shape, size, and crystallization, coalescence, and aggregation and stabilization processes (e.g., encamped coating of organics onto the metallic core of AgNP) of reduced silver crystalline, which is strictly correlated with different antimicrobial properties of AgNP (Pomastowski et al., 2016).

The bacteria contained in milk from the families $\mathrm{Lac}$ tococcus and Lactobacillus have a more positive effect on the restoration of the natural human bacterial flora. In addition to this, synthesized silver nanoparticles by LAB bacteria are proposed for treatment of various dis- eases and pathogens that can be found in dairy products as well. Moreover, the increasing ecological problems directs the researcher to continuously focus on sustainable development. This is the reason why the present research describes the outcomes and related discussions regarding AgNC synthesized by the safe Lactobacillus paracasei strain isolated from the dairy waste (whey). Additionally, it is essential to underline that the results included in this paper prove efficient synthesis $(75 \% \pm$ 4.02) and present in detail the physicochemical characteristics of the bioactive silver composites mediated by the safe strain as an eco-friendly, inexpensive, accessible precursor for biosilver composite mediation. Moreover, synthesized AgNC have shown antimicrobial effects against clinical pathogens such as $P$. mirabilis, A. baumannii, and $P$. aeruginosa. The purpose of the biological approach is to receive active biocomplexes that con- 
stitute first a solution for the problem associated with the infected dairy products and, second, an alternative to the phenomenon of drug-resistant bacteria and the problems associated with the result of drug resistance mechanisms (Prakasham et al., 2014). Therefore, Lactobacillus paracasei ssp. paracasei, isolated from whey, is certainly a potential safe source, given that it meets these requirements and the synthesized safe $\mathrm{AgNC}$, an alternative to existing antimicrobial agents.

Moreover, oxidative stress is recognized as a major factor concerning the increasing development of chronic diseases and is explained as an imbalance between free radicals and antioxidants. Reactive oxygen species (ROS) are chemically reactive molecules containing oxygen that represent the free radicals. It is necessary to underline that ROS play a 2 -fold effect as both beneficial and toxic to the living system depending on the concentration (Cheng et al., 2010). At a moderate or minimalized level ROS have beneficial effects such as defense against pathogenic microorganisms, whereas at a higher concentration ROS generate oxidative stress, causing damage to biomolecules (Pham-Huy et al., 2008). The antioxidants serve to counter-balance the effect of oxidants. When an imbalance is present between the antioxidants and oxidants, the accumulated free radicals lead to vigorous damage to nucleic acids, proteins, and lipids in living organisms. Therefore, human health has to be protected against an eventual infection generated by the improper packaging and storage of food products. Such agents with both antioxidants and antimicrobial effect (e.g., biologically synthesized AgNC) can be used in food and food contact products as preservatives, for example, for the production of polymer embedded metal nanoparticles for baby milk bottles and food containers for the storage of milk products (Carbone et al., 2016).

Moreover, antimicrobial and antioxidant effects of synthesized AgNC can ensure good packaging and preservation of the products with a synergistic effect against free radicals (e.g., ROS). Therefore, blocking the generation of free radicals by supplementation of antioxidants (e.g., silver NC) can have a beneficial role in preventing free radical-related diseases caused by inappropriate preservation. On the other hand, the incorrect processing or storage of dairy products can be a source of microorganisms (e.g., P. mirabilis, A. baumannii, and $P$. aeruginosa) extremely harmful to human health. In this fact, the AgNC synthesized in this study by LAB strain as a source can be used as effective antimicrobial and antioxidant agent in dairy sector, especially for food packaging material. Therefore, the present research will indeed shed further light on the discovery of an efficient, inexpensive, and not only safe bioactive agent giving rise to dairy and pharmaceutical applications (i.e., drug-resistant bacteria), skin infections, and problems associated with the result of mold illness (e.g., problems with intestinal microflora), but also a safe source of bioactive silver mediation.

\section{CONCLUSIONS}

This work first demonstrated the use of whey as a source of Lactobacillus paracasei LPC20 (deposit no. B/00287), being eco-friendly and available for the biosilver NC synthesis, and second, highlighted the use of the synthesized $\mathrm{NC}$ against a variety of human and milk pathogens, including multidrug-resistant bacteria and skin infections. Moreover, the present research has proven that the silver nanoparticle core is coated by the organic layer consisting of the metabolites secreted naturally by the bacterial strain isolated from the whey such as iso-/leucine $\mathrm{C}$ cysteine (I/L-C), cysteine $\mathrm{C}$ iso-/leucine (Cys-I/L), amino acid i-types: asparaginesarginine-serine (iN-iR-IS), and amino acids: tryptophan $\mathrm{C}$ serine (W-S) sequences. The obtained results characterized silver NC as a complex structure with the presence of both metallic silver nanoparticles as well as the organic surface (secreted metabolites) naturally coating the silver core. The synthesized LBPC-AgNC have been found as a form of cubic crystalline structure with nanoparticles size of average equal to $18 \pm 2.4 \mathrm{~nm}$ and $\mathrm{d}$-spacing of $0.2394 \mathrm{~nm}$. Besides the already mentioned data, the present work has also specified the sightings noticed 1- $\left([\mathrm{Ag}]^{+}\right), 2-\left([\mathrm{Ag} 2]^{+}\right)$, and multi- $\left([\mathrm{Ag} 13]^{+}\right.$, $\left.[\mathrm{Ag} 16]^{+}\right)$clusters with a different natural coated organic surface: $[\mathrm{Ag} 7-\mathrm{I} / \mathrm{L}-\mathrm{C}]^{+},[\mathrm{Ag} 13-\mathrm{Cys}-\mathrm{I} / \mathrm{L}]^{+},[\mathrm{Ag} 16-\mathrm{iN}-\mathrm{iR}-$ $\mathrm{IS}]^{+}$, and $[\mathrm{Ag} 17-\mathrm{W}-\mathrm{S}]^{+}$. The LBPC-AgNC has proven to be more effective and nontoxic than antibiotics alone given that it did not manifest antimicrobial potential to LAB strains. Moreover, the LBPC-AgNC have also shown a high antiradical effect displaying the ability to reduce the DPPH radical in a ratio of 1:1.

\section{ACKNOWLEDGMENTS}

This work was supported by the Opus 14 No. 2017/27/B/ST4/02628 (2018-2021) from the National Science Centre, Poland. B.B. and P.P. are members of Torun Center of Excellence "Towards Personalized Medicine" operating under Excellence Initiative-Research University (Poland). The authors declare that they have no conflict of interest.

\section{REFERENCES}

Abd-Elnaby, H. M., G. M. Abo-Elala, U. M. Abdel-Raouf, and M. M. Hamed. 2016. Antibacterial and anticancer activity of extracellular synthesized silver nanoparticles from marine Streptomyces rochei 
MHM13. Egypt. J. Aquat. Res. 42:301-312. https://doi.org/10 $.1016 /$ j.ejar.2016.05.004.

Adebayo-Tayo, B. C., and A. O. Popoola. 2017. Biogenic synthesis and antimicrobial activity of silver nanoparticle using exopolysaccharides from lactic acid bacteria. Int. J. Nanodimens. 8:61-69. https: //doi.org/10.22034/ijnd.2017.24377.

Awwad, M. A., M. N. Salem, and A. O. Abdeen. 2012. Biosynthesis of silver sanoparticles using Olea europaea leaves extract and its antibacterial activity. Nanosci. Nanotech. 2:164-170. https://doi .org/10.5923/j.nn.20120206.03.

Barth, A., and C. Zscherp. 2002. What vibrations tell us about proteins. Q. Rev. Biophys. 35:369-430. https://doi.org/10.1017/ S0033583502003815.

Bhakya, S., S. Muthukrishnan, M. Sukumaran, and M. Muthukumar. 2016. Biogenic synthesis of silver nanoparticles and their antioxidant and antibacterial activity. Appl. Nanosci. 6:755-766. https:// doi.org/10.1007/s13204-015-0473-z.

Bharathi, D., and V. Bhuvaneshwari. 2019. Evaluation of the cytotoxic and antioxidant activity of phyto-synthesized silver nanoparticles using Cassia angustifolia flowers. Biol. Nano. Sci. 9:155-163. https: //doi.org/10.1007/s12668-018-0577-5.

Bhat, R., R. Deshpande, S. V. Ganachari, D. S. Huh, and A. Venkataraman. 2011. Photo-irradiated biosynthesis of silver nanoparticles using edible mushroom pleurotus Florida and their antibacterial activity studies. Bioinorg. Chem. Appl. 2011:650979. https://doi .org $/ 10.1155 / 2011 / 650979$.

Blois, M. 1958. Antioxidant determinations by the use of a stable free radical. Nature 181:1199-1200. https://doi.org/10.1038/ $1811199 \mathrm{a} 0$

Bonnet, R., H. Marchandin, C. Chanal, D. Sirot, R. Labia, C. De Champs, E. Jumas-Bilak, and J. Sirot. 2002. Chromosome-encoded class D betalactamase OXA-23 in Proteus mirabilis. Antimicrob. Agents Chemother. 46:2004-2006. https://doi.org/10.1128/ AAC.46.6.2004-2006.2002.

Brand-Williams, W., M. E. Cuvelier, and C. Berset. 1995. Use of a free radical method to evaluate antioxidant activity. Lebensm. Wiss. Technol. 28:25-30. https://doi.org/10.1016/S0023-6438(95)80008 -5 .

Bruno, R. G. S., H. M. Rutigliano, R. L. Cerri, P. H. Robinson, and J. E. P. Santos. 2009. Effect of feeding Saccharomyces cerevisiae on performance of dairy cows during summer heat stress. Anim. Feed Sci. Technol. 150:175-186. https://doi.org/10.1016/j.anifeedsci 2008.09.001.

Buszewski, B., V. Railean-Plugaru, P. Pomastowski, K. Rafińska, M. Szultka-Mlynska, P. Golinska, M. Wypij, D. Laskowski, and H. Dahm. 2018. Antimicrobial activity of biosilver nanoparticles produced by a novel Streptacidiphilus durhamensis strain. J. Microbiol. Immunol. Infect. 51:45-54. https://doi.org/10.1016/j.jmii .2016.03.002.

Buszewski, B., V. Railean-Plugaru, P. Pomastowski, K. Rafinska, M. Szultka-Mlynska, and T. Kowalkowski. 2017. Antimicrobial effectiveness of bioactive silver nanoparticles synthesized by Actinomycetes HGG16n strain. Curr. Pharm. Biotechnol. 18:168-176. https: //doi.org/10.2174/1389201018666170104112434.

Carbone, M., D. T. Donia, G. Sabbatella, and R. Antiochia. 2016. Silver nanoparticles in polymeric matrices for fresh food packaging. J. King Saud Univ. Sci. 28:273-279. https://doi.org/10.1016/ j.jksus.2016.05.004.

Chauhan, R., A. J. Kumar, and S. Abraham. 2013. A biological approach to the synthesis of silver nanoparticles with Streptomyces sp JAR1 and its antimicrobial activity. Sci. Pharm. 81:607-621. https://doi.org/10.3797/scipharm.1302-02.

Cheng, L. X., J. J. Tang, H. Luo, X. L. Jin, F. Dai, J. Yang, Y. P. Qian, X. Z. Li, and B. Zhou. 2010. Antioxidant and antiproliferative activities of hydroxyl-substituted Schiff bases. Bioorg. Med. Chem. Lett. 20:2417-2420. https://doi.org/10.1016/j.bmcl.2010 .03.039.

Chernousova, S., and M. Epple. 2013. Silver as antibacterial agent: Ion, nanoparticle, and metal. Angew. Chem. Int. Ed. Engl. 52:1636-1653. https://doi.org/10.1002/anie.201205923.
Clinical and Laboratory Standards Institute. 2006. Performance standards for antimicrobial susceptibility testing. Supplement M100S16. Clinical and Laboratory Standards Institute, Wayne, PA.

Daphedar, A. 2020. Synthesis and characterization of Silver nanoparticles from fruit extract of Michelia champaca L.: Their antioxidant and antibacterial activity. Int. J. Nanodimens. 11:267-276.

Deng, H., D. McShan, Y. Zhang, S. S. Sinha, Z. Arslan, P. C. Ray, and H. Yu. 2016. Mechanistic study of the synergistic antibacterial activity of combined silver nanoparticles and common antibiotics. Environ. Sci. Technol. 50:8840-8848. https://doi.org/10.1021/acs .est.6b00998.

Dhanashekar, R., S. Akkinepalli, and A. Nellutla. 2012. Milk-borne infections. An analysis of their potential effect on the milk industry. Germs 2:101-109. https://doi.org/10.11599/germs.2012.1020.

Dibrov, P., J. Dzioba, K. Gosink, and C. C. Hase. 2002. Chemiosmotic mechanism of antimicrobial activity of $\mathrm{Ag}+$ in Vibrio cholerae. Antimicrob. Agents Chemother. 46:2668-2670. https://doi.org/10 .1128/AAC.46.8.2668-2670.2002.

Garmasheva, I., N. Kovalenko, S. Voychuk, A. Ostapchuk, O. Livins'ka, and L. Oleschenko. 2016. Lactobacillus species mediated synthesis of silver nanoparticles and their antibacterial activity against opportunistic pathogens in vitro. Bioimpacts 6:219-223. https://doi .org/10.15171/bi.2016.29.

Ghisaidoobe, A. B. T., and S. J. Chung. 2014. Intrinsic tryptophan fluorescence in the detection and analysis of proteins: A focus on Förster resonance energy transfer techniques. Int. J. Mol. Sci. 15:22518-22538. https://doi.org/10.3390/ijms151222518.

Hassan, A. N., and J. F. Frank. 2011. Microorganisms associated with milk. Encyclopedia of Dairy Sciences. 2:447-457.

Hsiao, I. L., Y. K. Hsieh, C. F. Wang, I. C. Chen, and Y. J. Huang. 2015. Trojan-horse mechanism in the cellular uptake of silver nanoparticles verified by direct intra- and extracellular silver speciation analysis. Environ. Sci. Technol. 49:3813-3821. https://doi .org/10.1021/es504705p.

Hunter, R. J. 1988. Zeta Potential in Colloid Science. Principles and Applications. Academic Press, London, UK.

Jung, Z. 1926. Kristallogr., Kristallgeom., Kristallphys. Kristallchem. 64:422. (ref. no. 00-003-0921). X'Pert Pro Analytical diffractometer database (Phillips, Erlangen, Germany).

Khorrami, S., A. Zarrabi, M. Khaleghi, M. Danaei, and M. R. Mozafari. 2018. Selective cytotoxicity of green synthesized silver nanoparticles against the MCF-7 tumor cell line and their enhanced antioxidant and antimicrobial properties. Int. J. Nanomedicine 13:8013-8024. https://doi.org/10.2147/IJN.S189295.

Lok, C. N., C. M. Ho, R. Chen, Q. Y. He, W. Y. Yu, H. Sun, P. K. H. Tam, J. F. Chiu, and C. M. Che. 2006. Proteomic analysis of the mode of antibacterial action of silver nanoparticles. J. Proteome Res. 5:916-924. https://doi.org/10.1021/pr0504079.

Mishra, K., H. Ojha, and N. K. Chaudhury. 2012. Estimation of antiradical properties of antioxidants using DPPH assay: Critical review and results. Food Chem. 130:1036-1043. https://doi.org/10 .1016/j.foodchem.2011.07.127.

Mohanta, Y. K., and S. K. Behera. 2014. Biosynthesis, characterization and antimicrobial activity of silver nanoparticles by Streptomyces sp. SS2. Bioprocess Biosyst. Eng. 37:2263-2269. https://doi .org/10.1007/s00449-014-1205-6.

Movasaghi, Z., S. Rehman, and I. Rehman. 2008. Fourier transform infrared (FTIR) spectroscopy of biological tissues. Appl. Spectrosc. Rev. 43:134-179. https://doi.org/10.1080/05704920701829043.

Pham-Huy, L. A., H. Hua, and C. Pham-Huy. 2008. Free radicals, antioxidants in disease and health. Int. J. Biomed. Sci. 4:89-96.

Poirel, L., G. F. Weldhagen, T. Naas, C. De Champs, M. G. Dove, and P. Nordmann. 2001. GES-2, a class A beta-lactamase from Pseudomonas aeruginosa with increased hydrolysis of imipenem. Antimicrob. Agents Chemother. 45:2598-2603. https://doi.org/10 .1128/AAC.45.9.2598-2603.2001.

Pomastowski, P., and B. Buszewski. 2019. Complementarity of matrix- and nanostructure-assisted laser desorption/ionization approaches. Nanomaterials (Basel) 9:260. https://doi.org/10.3390/ nano9020260. 
Pomastowski, P., M. Sprynskyy, P. Žuvela, K. Rafińska, M. Milanowski, J. J. Liu, M. Yi, and B. Buszewski. 2016. Silver-lactoferrin nanocomplexes as a potent antimicrobial agent. J. Am. Chem. Soc. 138:7899-7909. https://doi.org/10.1021/jacs.6b02699.

Pomastowski, P., M. Szultka, W. Kupczyk, M. Jackowski, and B. Buszewski. 2015. Evaluation of intact cell matrix-assisted laser desorption/ionization time-of-flight mass spectrometry for capillary electrophoresis detection of controlled bacterial clumping. J. Anal. Bioanal. Tech. S13:008. https://doi.org/10.4172/2155-9872 S13-008.

Pomastowski, P., M. Złoch, A. Rodzik, M. Ligor, M. Kostrzewa, and B. Buszewski. 2019. Analysis of bacteria associated with honeys of different geographical and botanical origin using two different identification approaches: MALDI-TOF MS and $16 \mathrm{~S}$ rDNA PCR technique. PLoS One 14:e0217078. https://doi.org/10.1371/ journal.pone.0217078.

Prabhu, S., and E. K. Poulose. 2012. Silver nanoparticles: Mechanism of antimicrobial action, synthesis, medical applications, and toxicity effects. Int. Nano Lett. 2:32. https://doi.org/10.1186/2228 $-5326-2-32$.

Prakasham, R. S., B. S. Kumar, Y. S. Kumar, and K. P. Kumar. 2014. Production and characterization of protein encapsulated silver nanoparticles by marine isolate Streptomyces parvulus ssnp11. Indian J. Microbiol. 54:329-336. https://doi.org/10.1007/s12088 -014-0452-1.

Railean-Plugaru, V., P. Pomastowski, T. Kowalkowski, M. Sprynskyy, and B. Buszewski. 2018. Physicochemical study of natural fractionated biocolloid by asymmetric flow field-flow fractionation in tandem with various complementary techniques using biologically synthesized silver nanocomposites. Anal. Bioanal. Chem. 410:2837-2847. https://doi.org/10.1007/s00216-018-0967-0.

Railean-Plugaru, V., P. Pomastowski, K. Meller, M. Zloch, K. Rafinska, and B. Buszewski. 2017. Lactococcus lactis as a safe and inexpensive source of bioactive silver composites. Appl. Microbiol. Biotechnol. 101:7141-7153. https://doi.org/10.1007/s00253-017 $-8443-\mathrm{x}$.
Railean-Plugaru, V., P. Pomastowski, K. Rafinska, M. Wypij, W. Kupczyk, H. Dahm, M. Jackowski, and B. Buszewski. 2016a. Antimicrobial properties of biosynthesized silver nanoparticles studied by flow cytometry and related techniques. Electrophoresis 37:752761. https://doi.org/10.1002/elps.201500507.

Railean-Plugaru, V., P. Pomastowski, M. Wypij, M. Szultka-Mlynska, K. Rafinska, P. Golinska, H. Dahm, and B. Buszewski. 2016b. Study of silver nanoparticles synthesized by acidophilic strain of Actinobacteria isolated from the of Picea sitchensis forest soil. J. Appl. Microbiol. 120:1250-1263. https://doi.org/10.1111/jam .13093 .

Rogowska, A., K. Rafińska, P. Pomastowski, J. Walczak, V. RaileanPlugaru, M. Buszewska-Forajta, and B. Buszewski. 2017. Silver nanoparticles functionalized with ampicillin. Electrophoresis 38:2757-2764. https://doi.org/10.1002/elps.201700093.

Saad, N. M., W. Amin, and S. Mostafa. 2018. Detection of Acinetobacter species in milk and some dairy products. Assiut Vet. Med. J. $64: 34-40$.

Salari, S., S. Esmaeilzadeh Bahabadi, A. Samzadeh-Kermani, and F. Yosefzaei. 2019. n-vitro evaluation of antioxidant and antibacterial potential of green synthesized silver nanoparticles using Prosopis farcta fruit extract. Iran. J. Pharm. Res. 18:430-455.

Singh, R., U. U. Shedbalkar, S. A. Wadhwani, and B. A. Chopade. 2015. Bacteriagenic silver nanoparticles: Synthesis, mechanism, and applications. Appl. Microbiol. Biotechnol. 99:4579-4593. https://doi.org/10.1007/s00253-015-6622-1.

Siwach, O. P., and P. Sen. 2009. Fluorescence properties of $\mathrm{Ag}$ nanoparticles in water, methanol and hexane. J. Lumin. 129:6-11. https://doi.org/10.1016/j.jlumin.2008.07.010.

Venyaminov, S. Y. U., and N. N. Kalnin. 1990. Quantitative IR spectrophotometry of peptide compounds in water solutions. I. Spectral parameters of amino acid residue absorption bands. Biopolymers 30:1243-1257. https://doi.org/10.1002/bip.360301309. 